\title{
Catalytic Asymmetric [2,3]-Sigmatropic Rearrangement of Sulfur Ylides generated from Copper(I) Carbenoids and Allyl Sulfides
}

\author{
Xiaomei Zhang, ${ }^{\mathrm{a}}$ Zhaohui Qu, ${ }^{\mathrm{a}}$ Zhihua Ma, ${ }^{\mathrm{a}}$ Weifeng Shi ${ }^{\mathrm{a}}$, Xianglin Jin ${ }^{\mathrm{a}}$ \\ and Jianbo Wang ${ }^{\mathrm{a}, \mathrm{b}_{*}}$
}

${ }^{a}$ Key Laboratory of Bioorganic Chemistry and Molecular Engineering of Ministry of Education, Department of Chemical Biology, College of Chemistry, Peking University, Beijing 100871, P. R. China,

${ }^{b}$ State Key Laboratory of Elemento-Organic Chemistry, Nankai University, Tianjin 300071, P. R. China

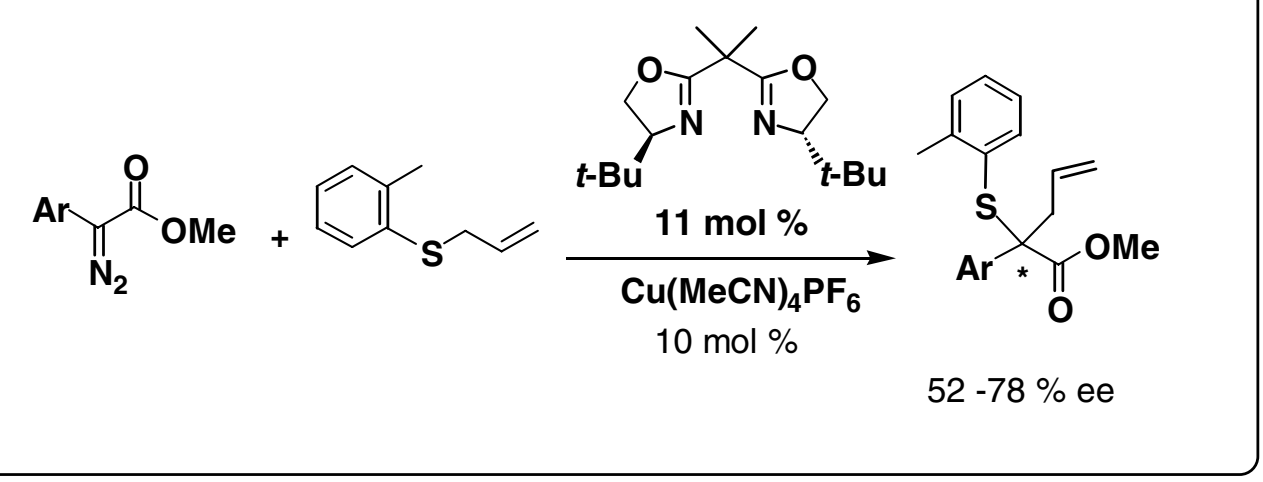


HPLC analysis was performed at HP 1100 apparatus.

$A r^{\prime}=P h, A r=P h$, Racemic (Hexane/iso-propanol 85:15)

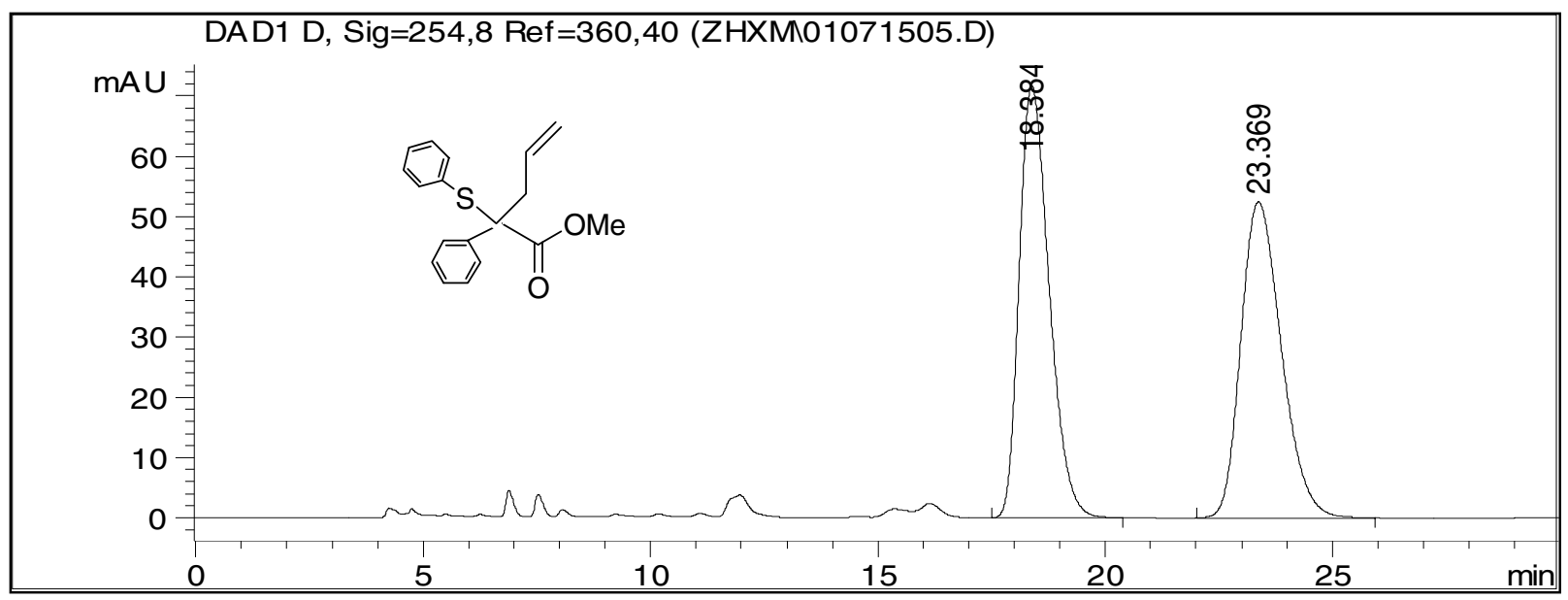

$\boldsymbol{A r} \boldsymbol{r}^{\prime}=\boldsymbol{P h}, \boldsymbol{A r}=\boldsymbol{P h}$, Table 1, Entry 1 (Hexane/iso-propanol 85:15)

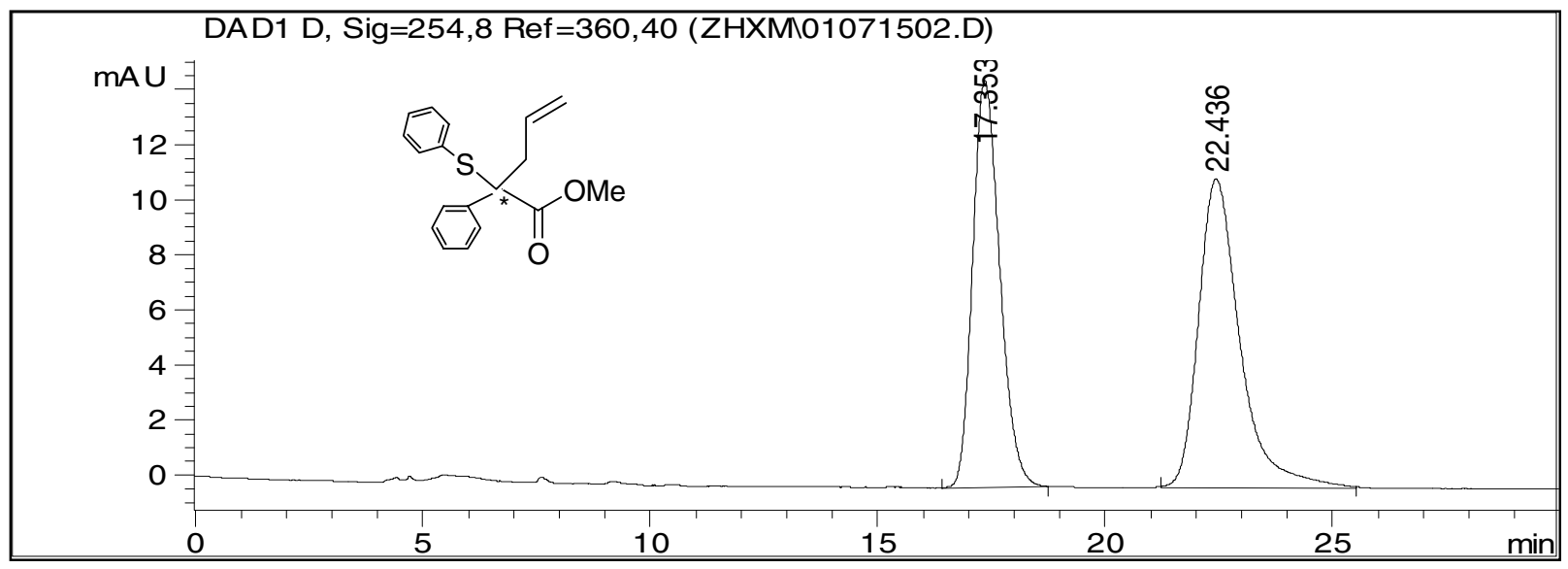

$A r^{\prime}=P h, A r=P h$, Table 1, Entry 2 (Hexane/iso-propanol 85:15)

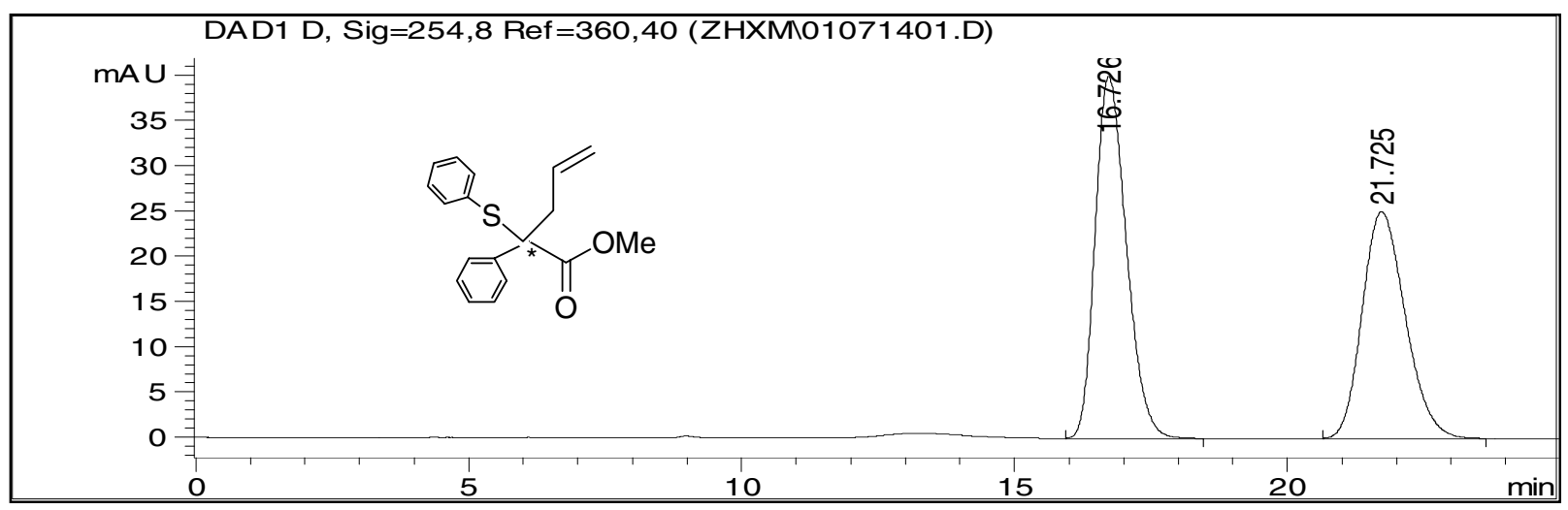


$A r^{\prime}=P h, A r=P h$, Table 1, Entry 3 (Hexane/iso-propanol 85:15)

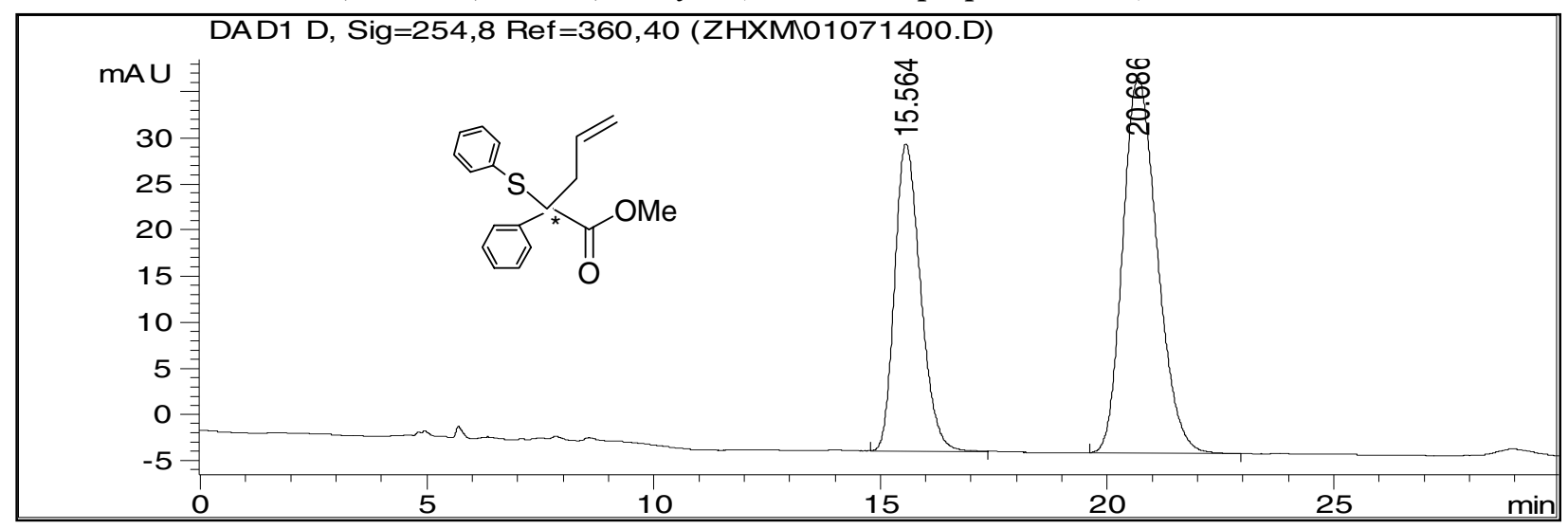

$A r^{\prime}=P h, A r=P h$, Table 1, Entry 7 (Hexane/iso-propanol 85:15)

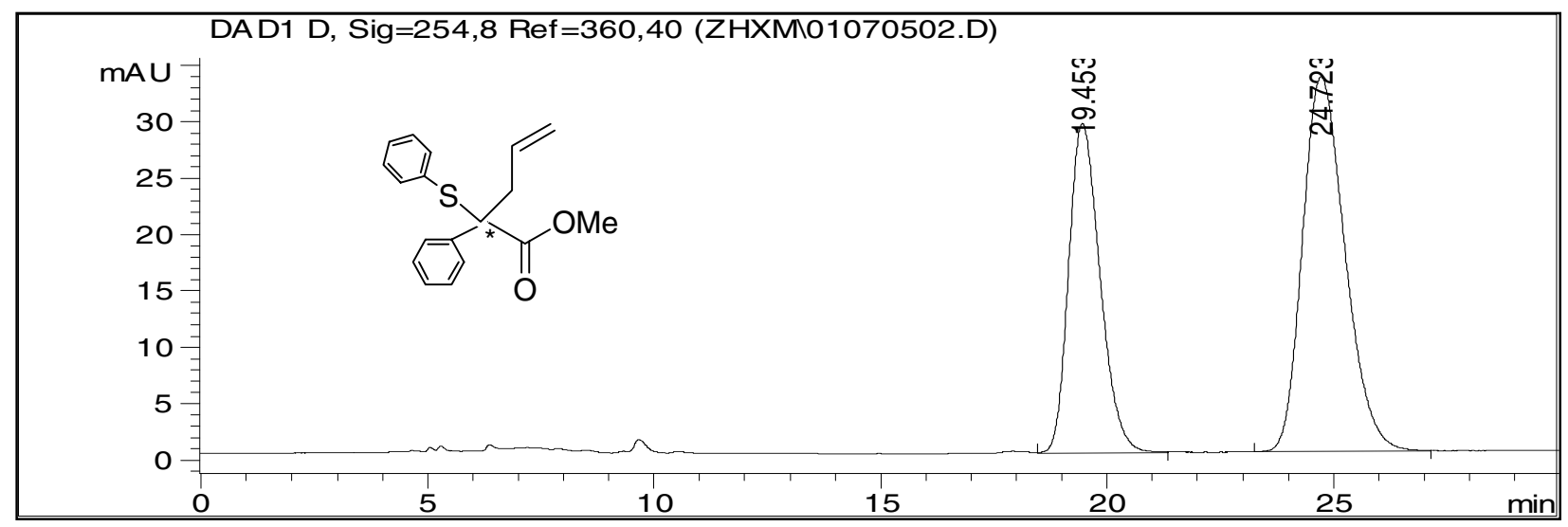

$A r^{\prime}=P h, A r=P h$, Table 1, Entry 11 (Hexane/iso-propanol 85:15)

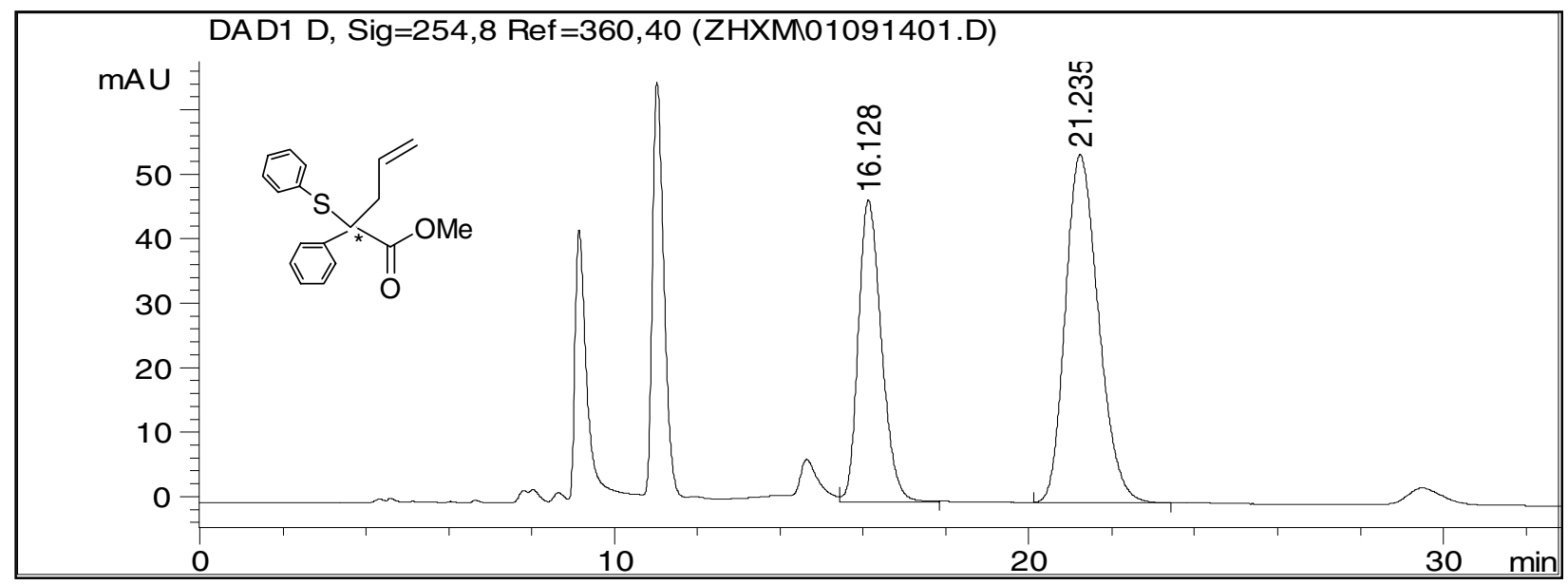


$A r^{\prime}=P h, A r=P h$, Racemic $\quad$ (Hexane/iso-propanol 90:10)

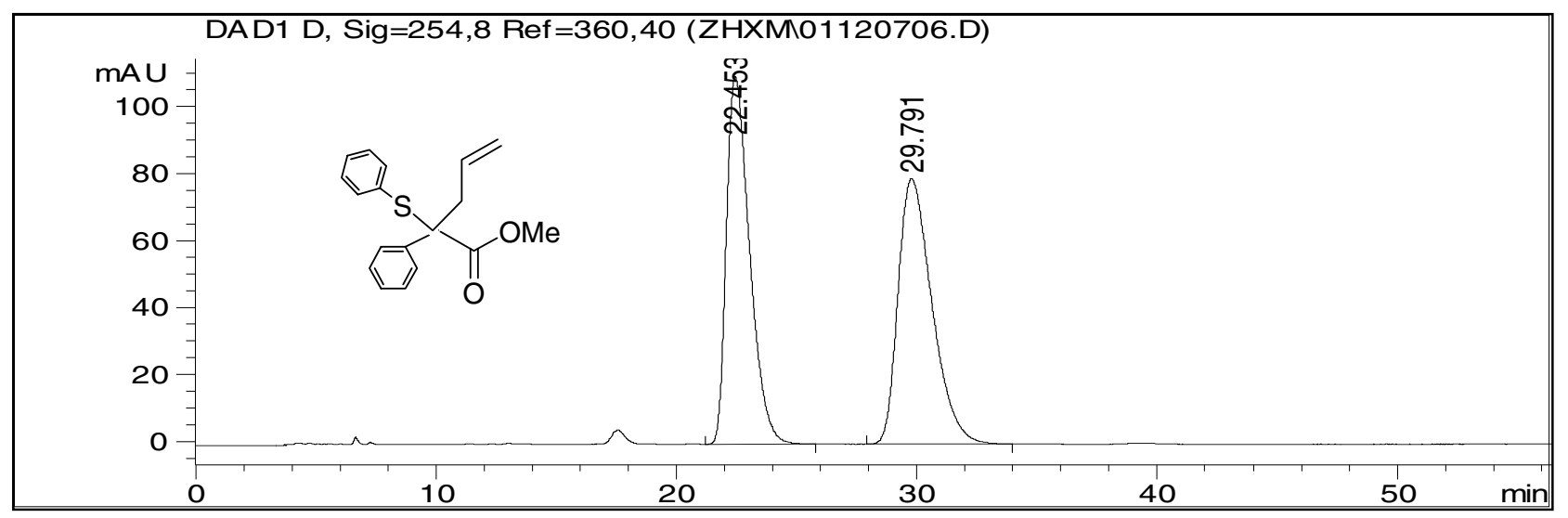

$A r^{\prime}=P h, A r=P h$, Table 1, Entry 16 (Hexane/iso-propanol 90:10)

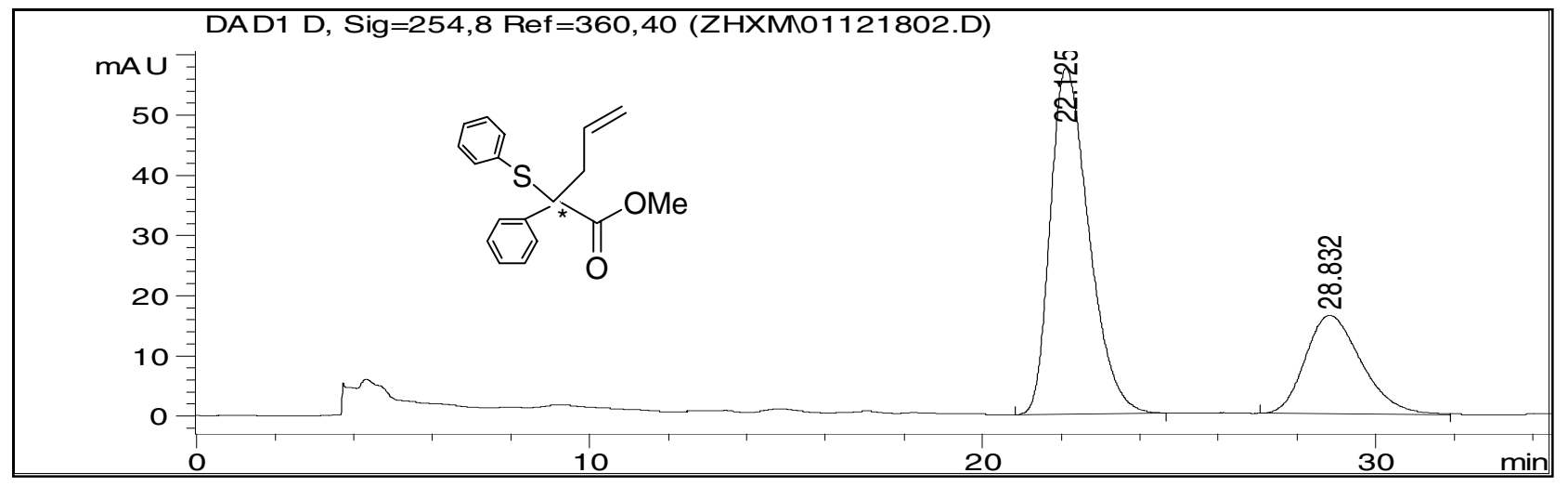

$A r '=2-M e P h, A r=P h$, Racemic (Hexane/iso-propanol 90:10)

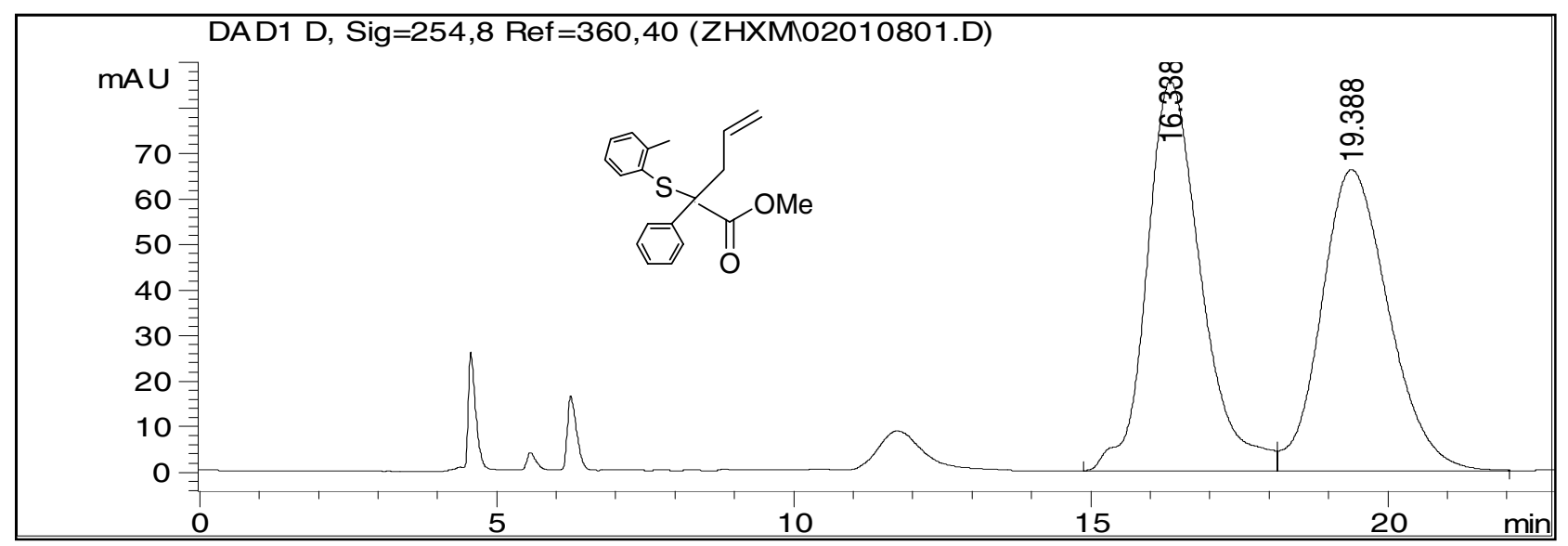


$A r^{\prime}=2-M e P h, A r=P h$, Table 1, Entry 4 (Hexane/iso-propanol 90:10)

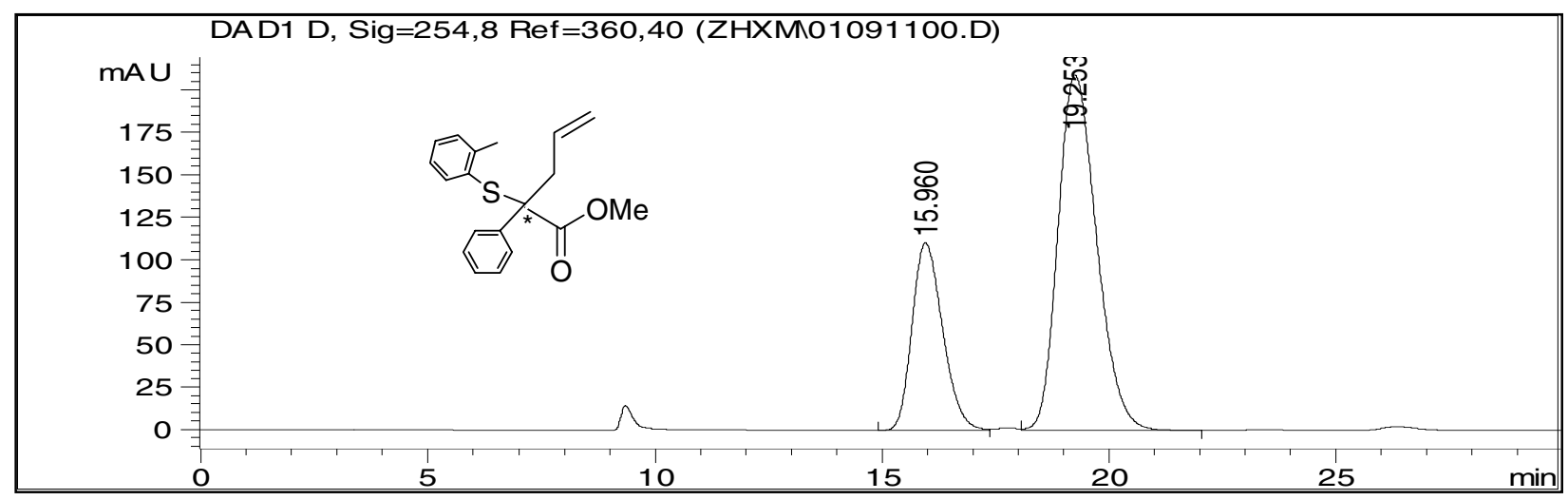

$A r^{\prime}=2-M e P h, A r=P h$, Table 1, Entry 5 (Hexane/iso-propanol 90:10)

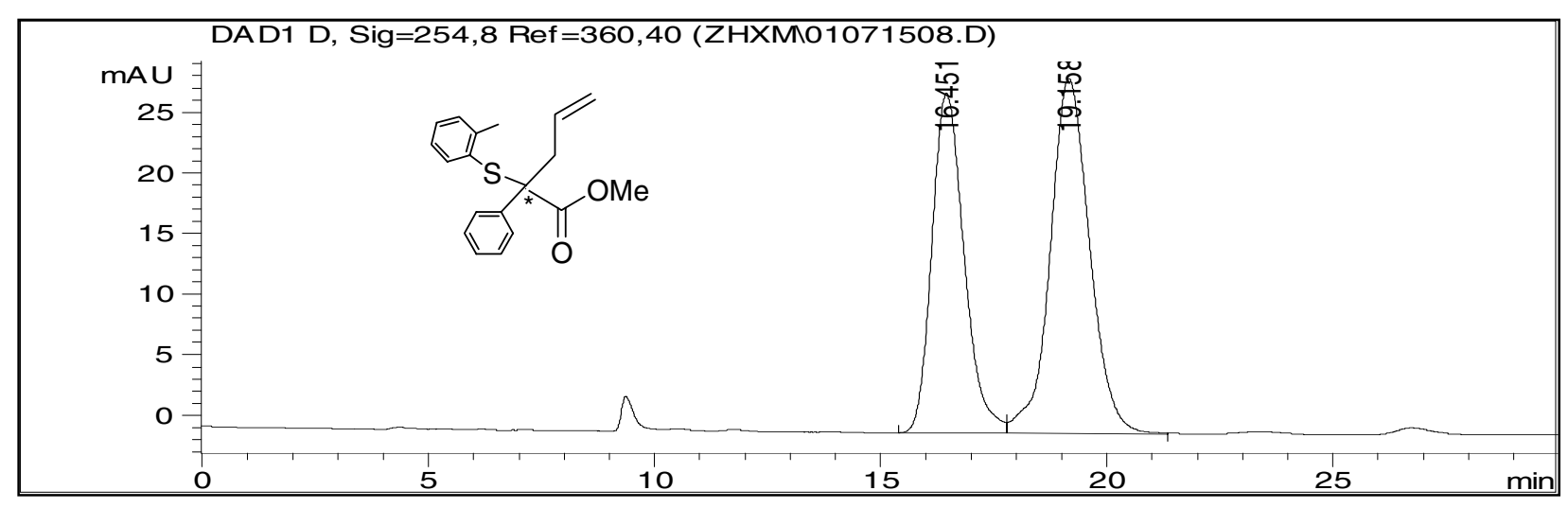

Ar' = 2-MePh, Ar =Ph, Table 1, Entry 6 (Hexane/iso-propanol 90:10)

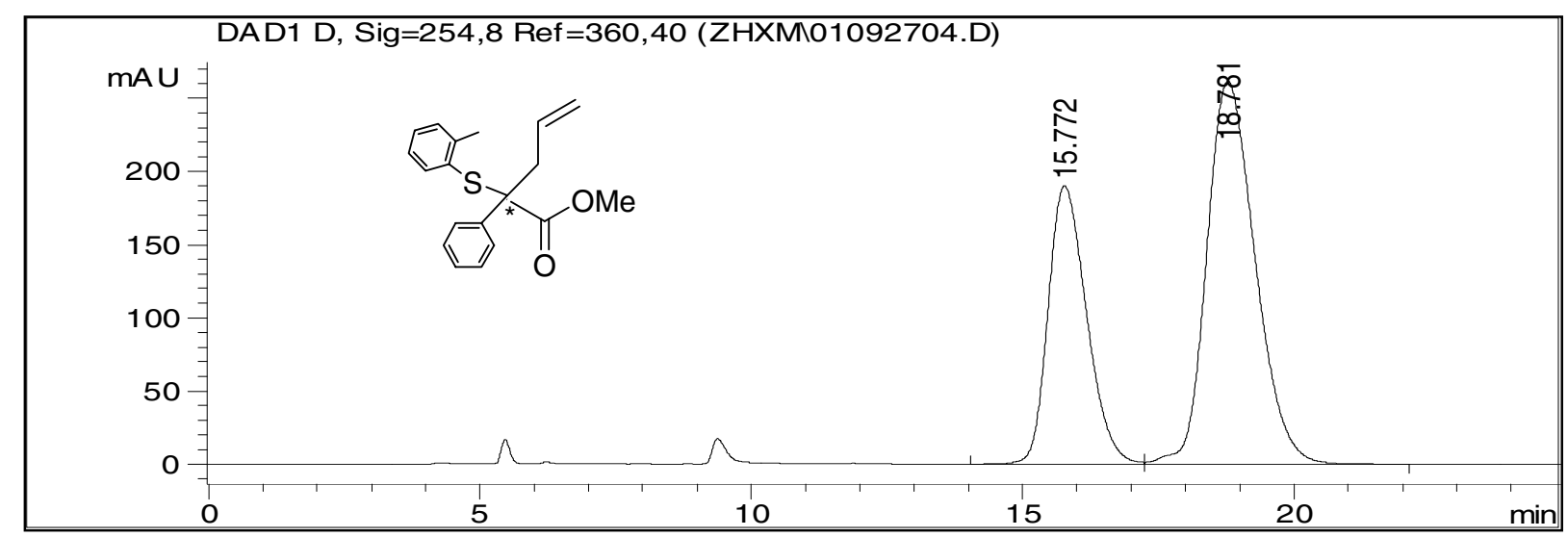


$A r^{\prime}=2-M e P h, A r=P h$, Table 1, Entry 10 (Hexane/iso-propanol 90:10)

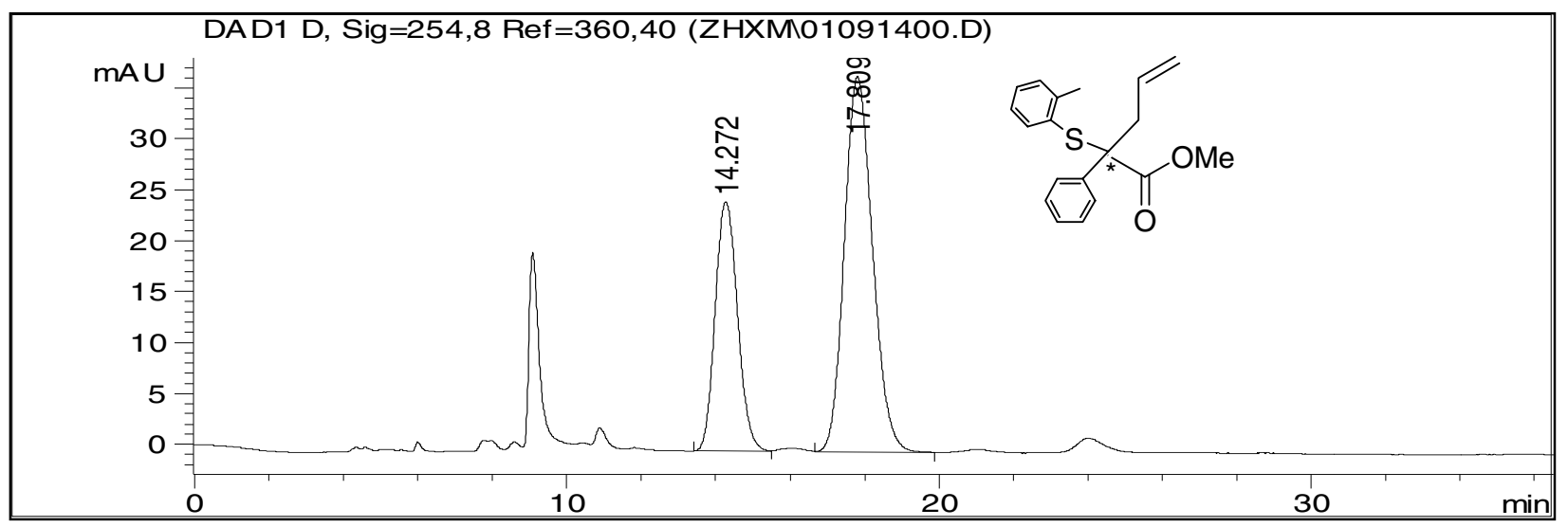

Ar' = 2-MePh, Ar = Ph, Table 1, Entry 14 (Hexane/iso-propanol 90:10)

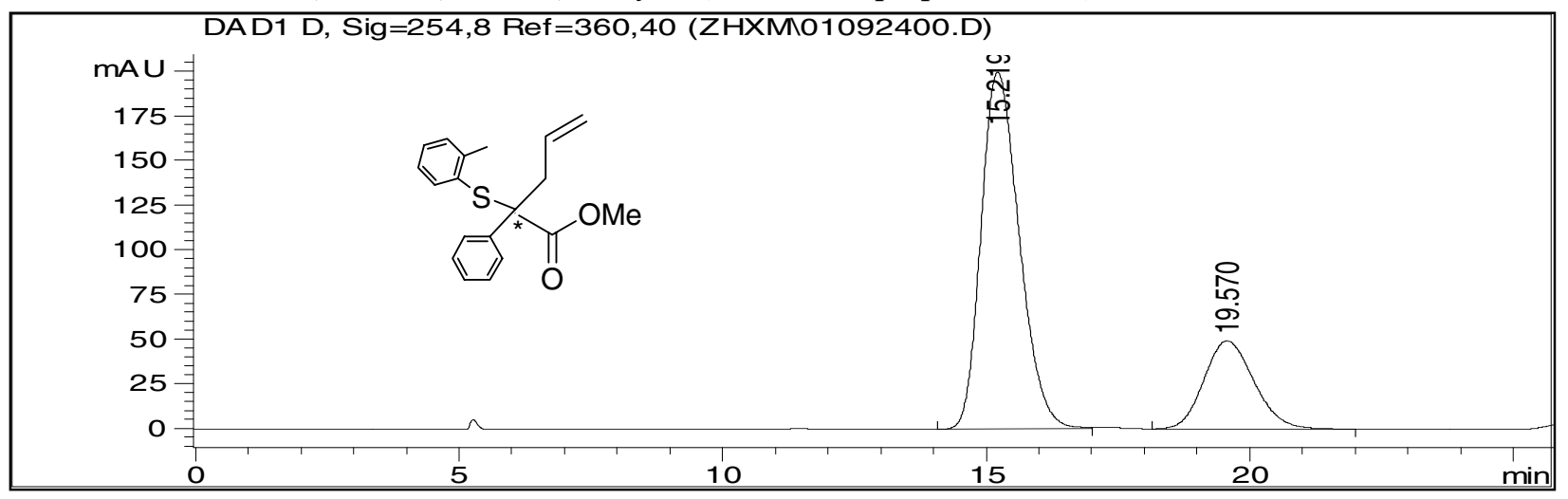

$A r^{\prime}=2-M e P h, A r=P h$, Table 1, Entry 17 (Hexane/iso-propanol 90:10)

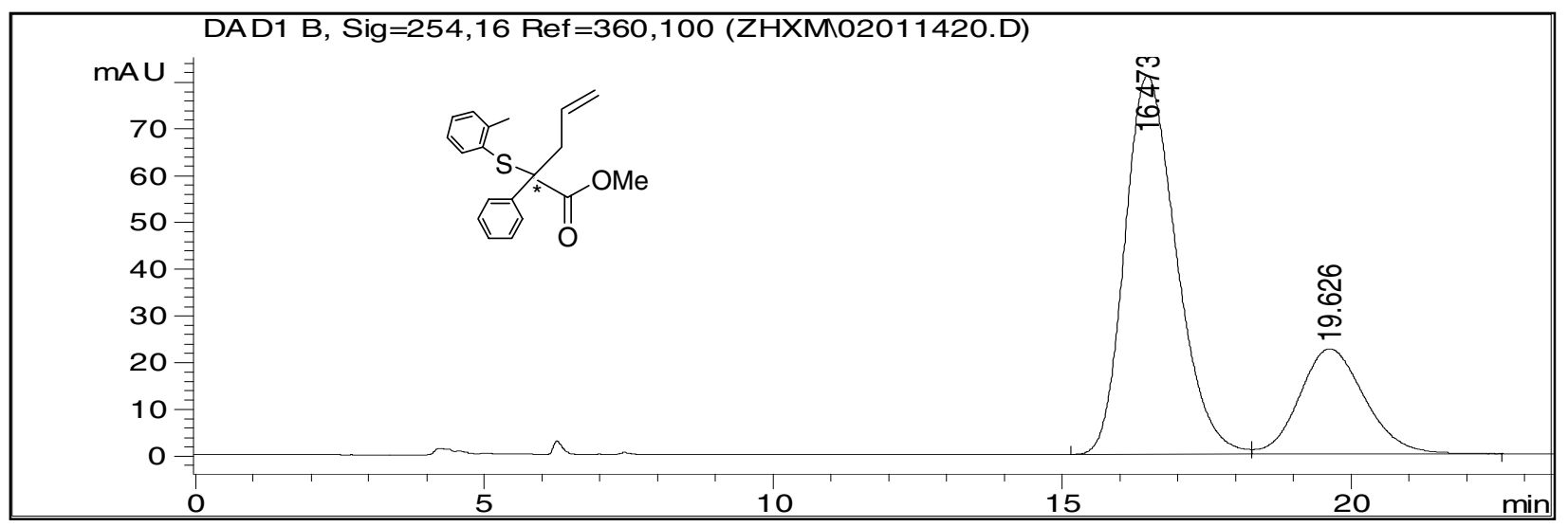


$A r^{\prime}=2-M e P h, A r=P h$, Table 1, Entry 18 (Hexane/iso-propanol 90:10)

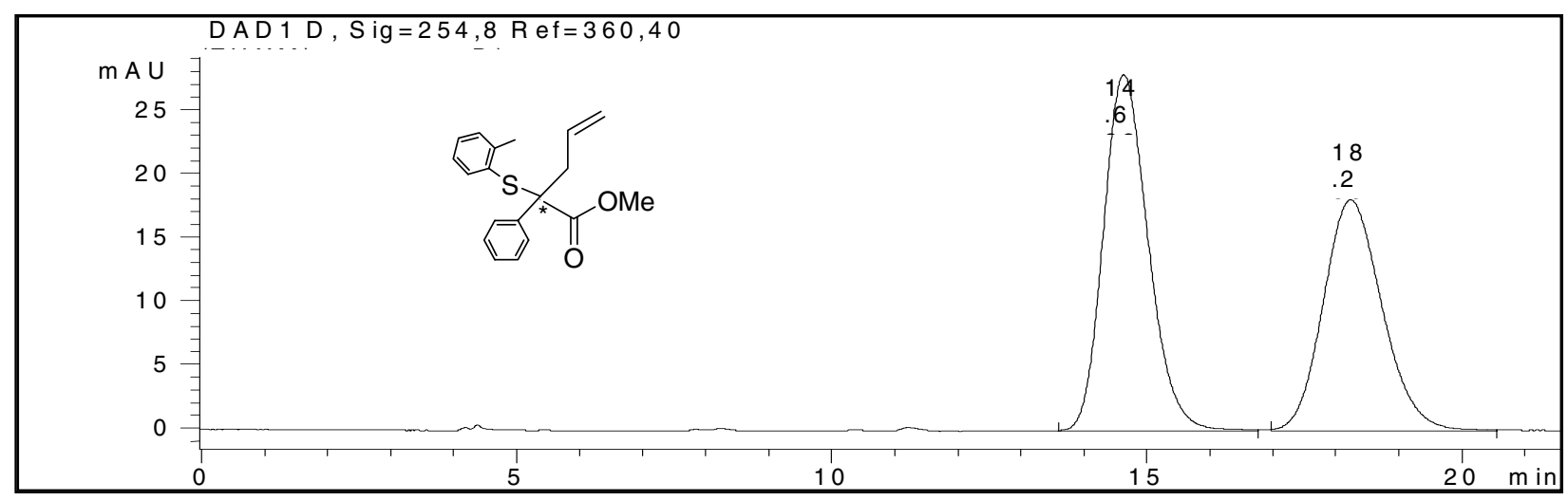

$A r^{\prime}=2-M e P h, A r=P h$, Table 1, Entry 19 (Hexane/iso-propanol 90:10)

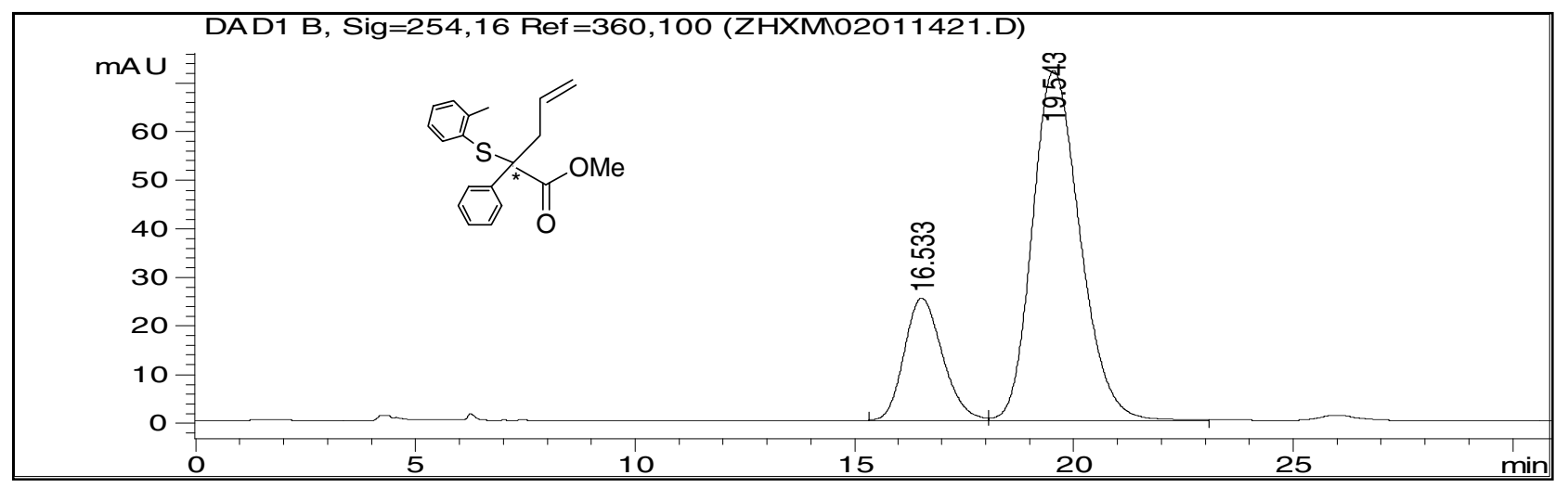

$A r^{\prime}=2-C l P h, A r=P h$, Racemic (Hexane/iso-propanol 90:10)

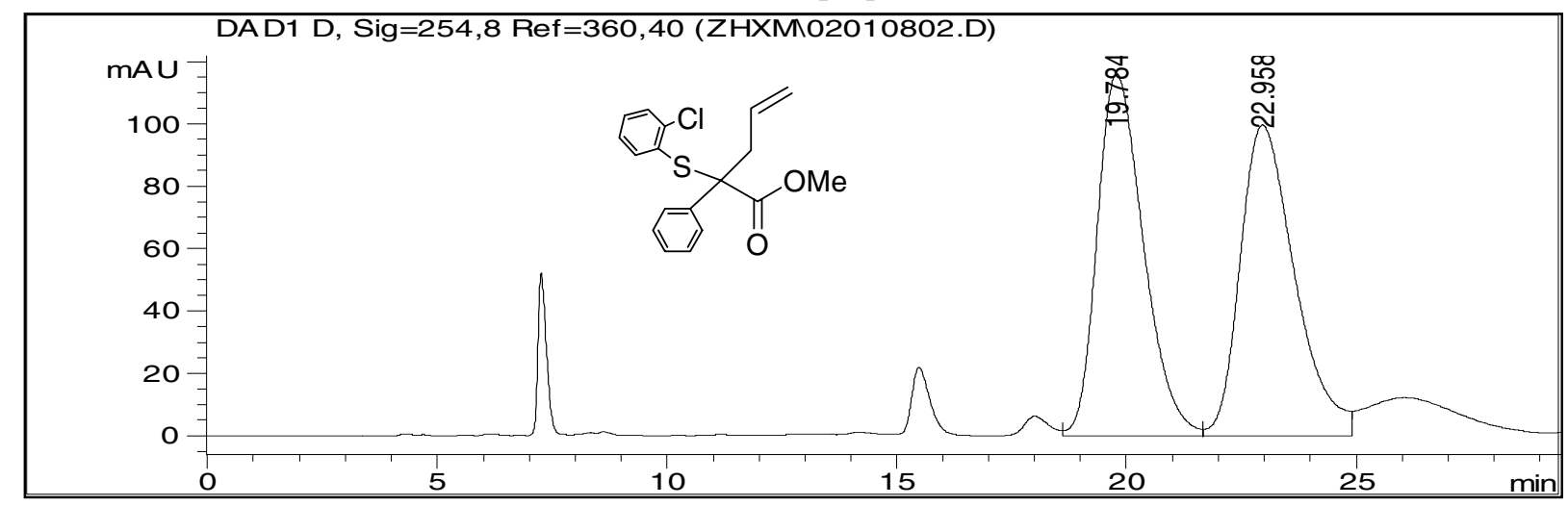


$A r^{\prime}=2-C l P h, A r=P h$, Table 1, Entry 8 (Hexane/iso-propanol 90:10)

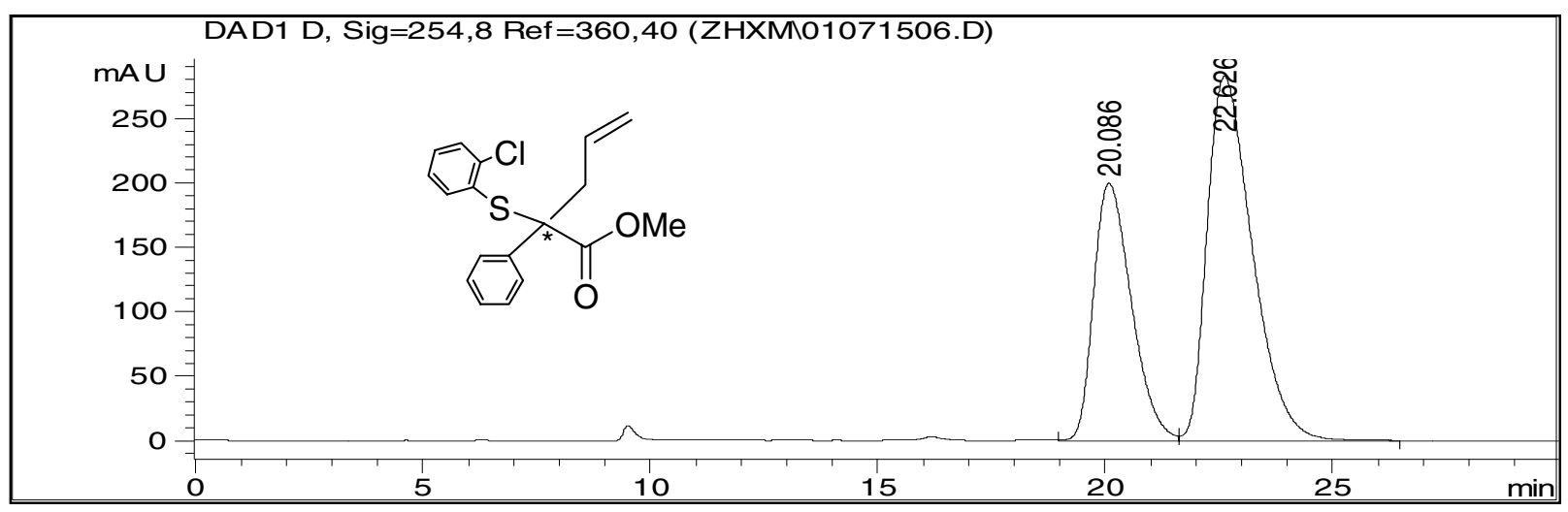

Ar' = 2-ClPh, Ar = Ph, Table 1, Entry 12 (Hexane/iso-propanol 90:10)

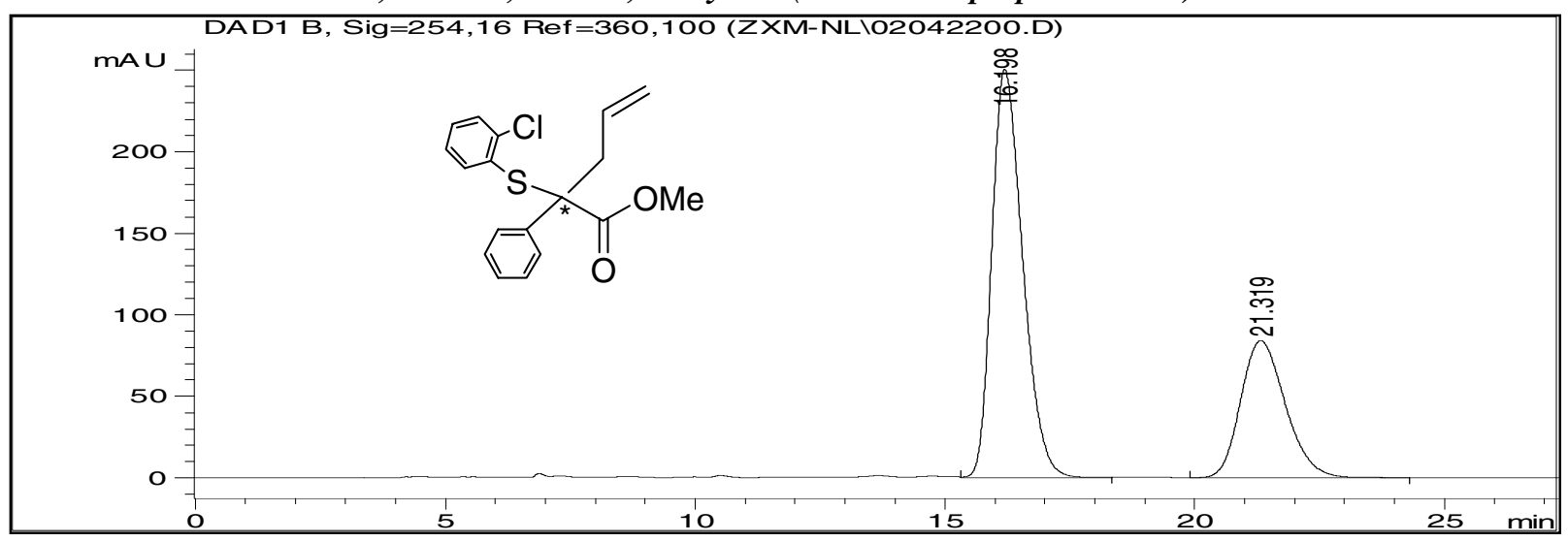

$A r^{\prime}=2,6-(\mathrm{Me})_{2} \mathrm{Ph}, \mathrm{Ar}=\mathrm{Ph}$, Racemic (Hexane/iso-propanol 90:10)

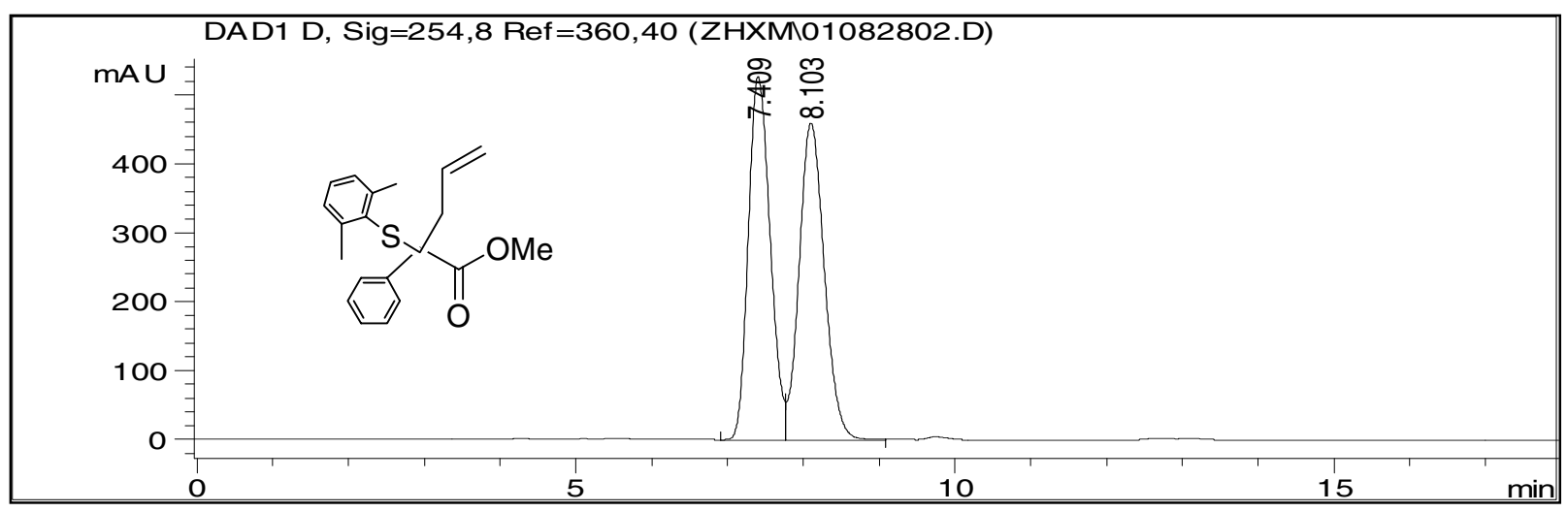


$A r^{\prime}=2,6-(\mathrm{Me})_{2} P h, A r=P h$, Table 1, Entry 9 (Hexane/iso-propanol 90:10)

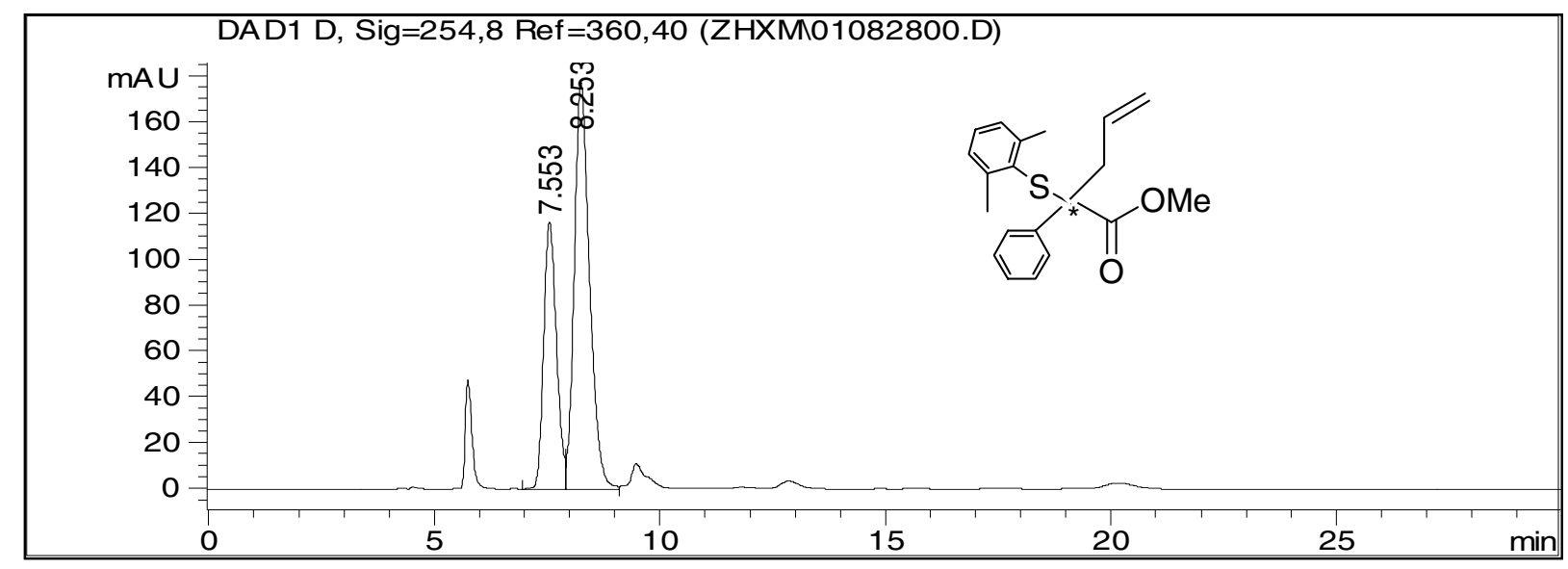

$A r^{\prime}=2-M e P h, A r=p-M e O P h$, Racemic (Hexane/iso-propanol 90:10)

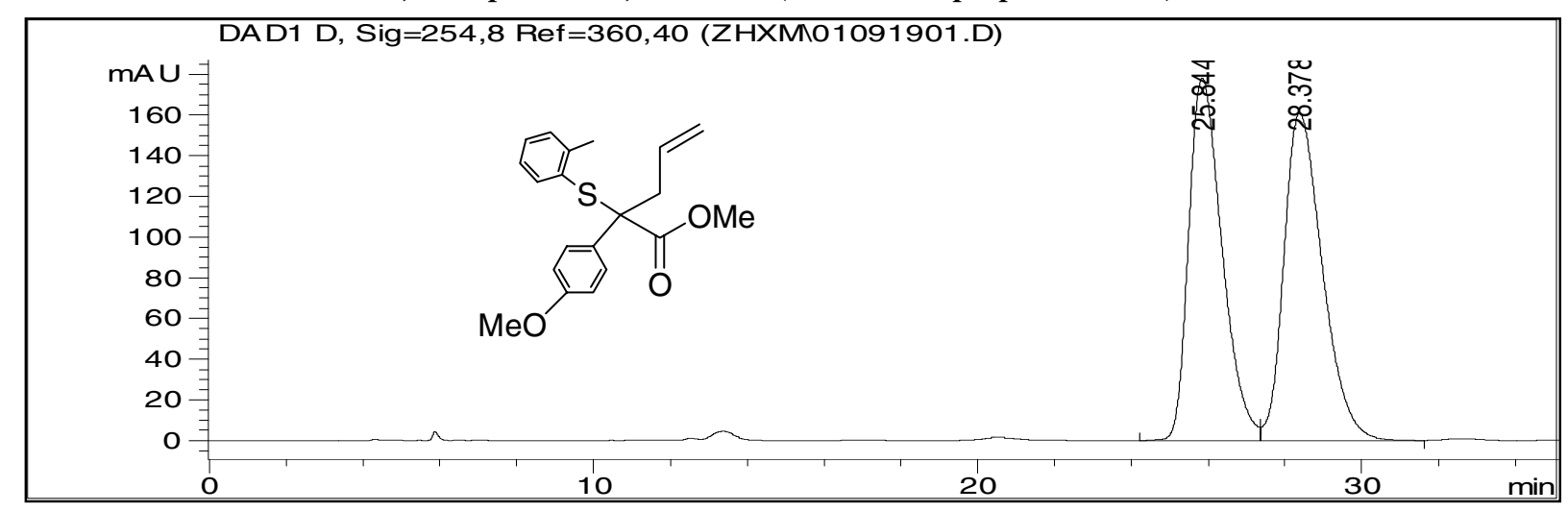

$A r^{\prime}=2-M e P h, A r=p-M e O P h$, Table 1, Entry 15 (Hexane/iso-propanol 90:10

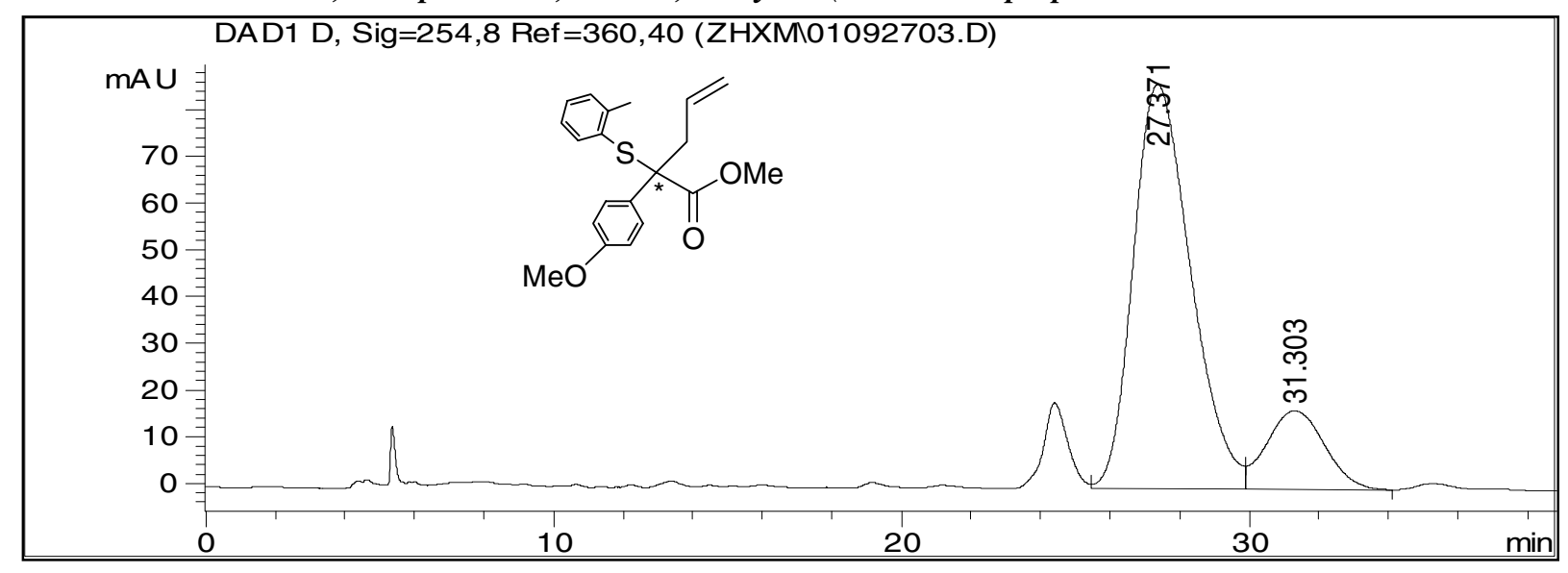


$A r^{\prime}=2-\mathrm{MePh}, A r=p-\mathrm{MeOPh}$, Table 2, Entry 3 (Hexane/iso-propanol 90:10)

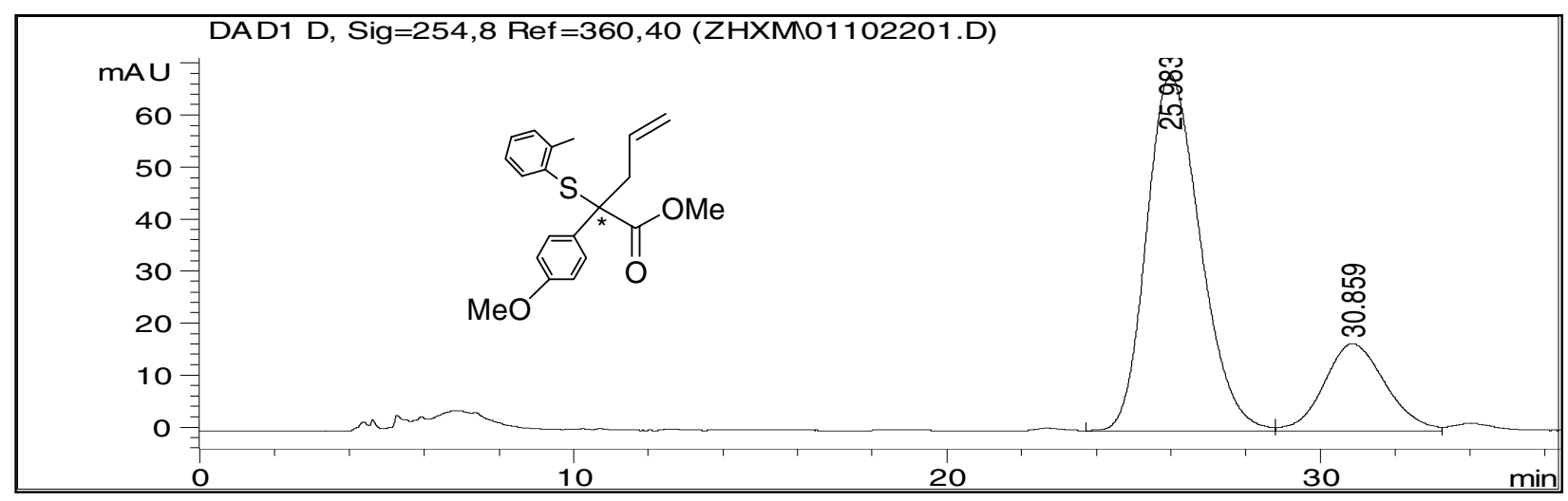

$A r^{\prime}=2-M e P h, A r=m-M e O P h$, Racemic (Hexane/iso-propanol 99:1)

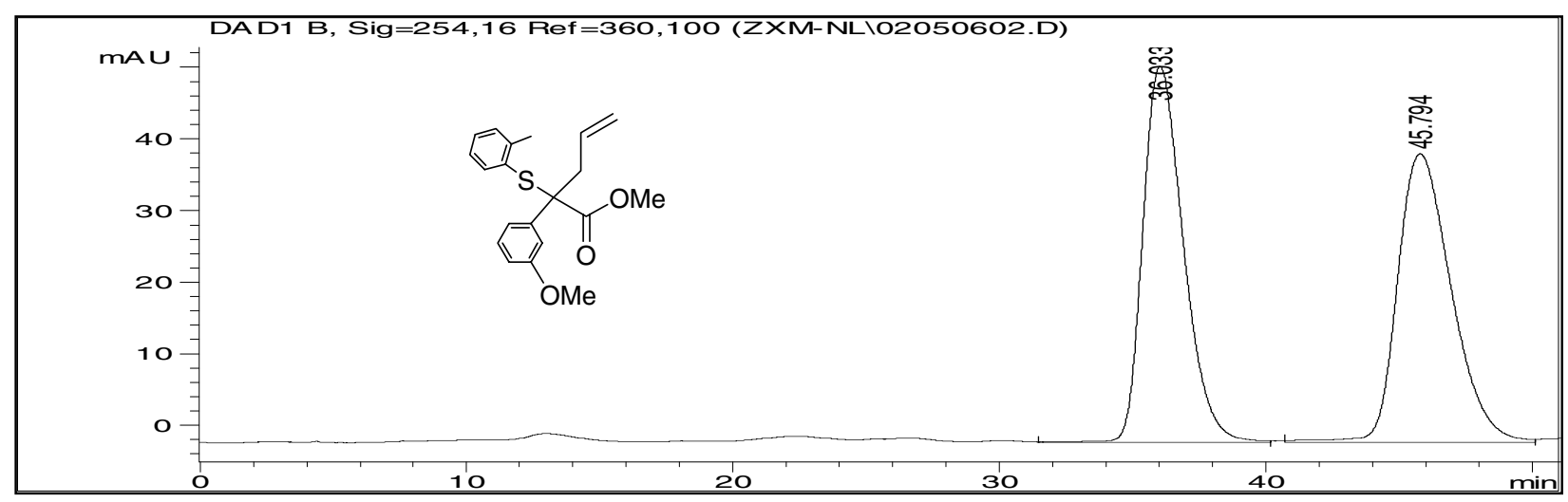

$A r^{\prime}=2-M e P h, A r=m-M e O P h$, Table 2, Entry 4 (Hexane/iso-propanol 99:1)

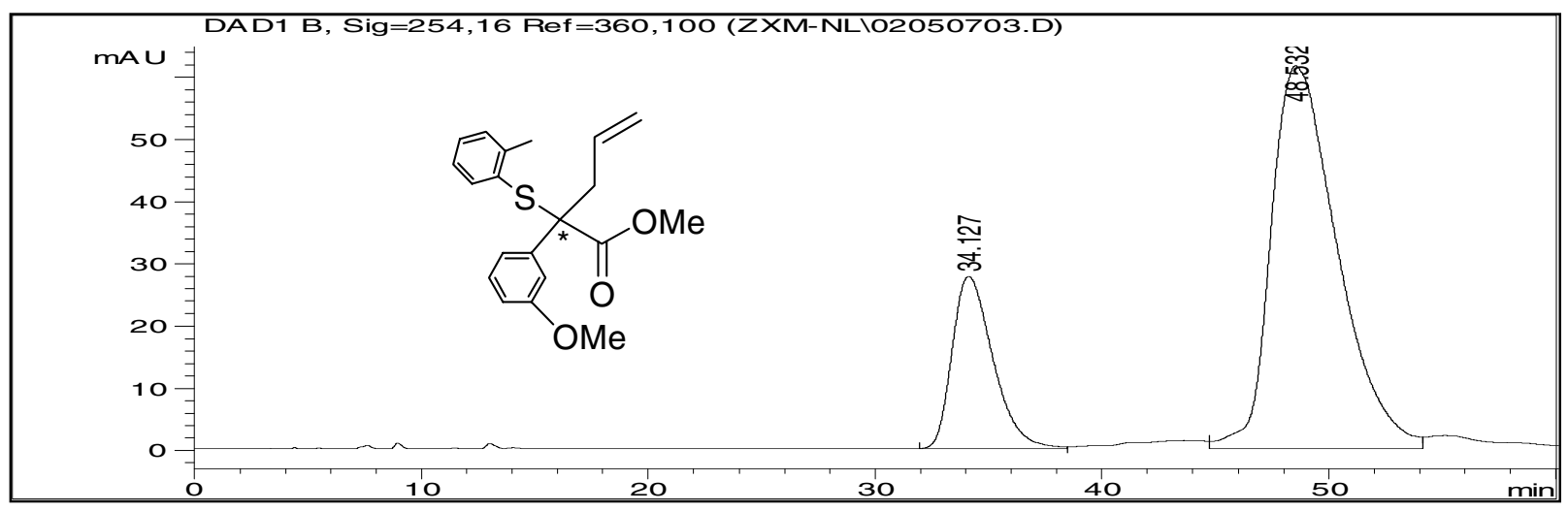


Ar' = 2-MePh, Ar = m-MeOPh, Table 2, Entry 5 (Hexane/iso-propanol 99:1)

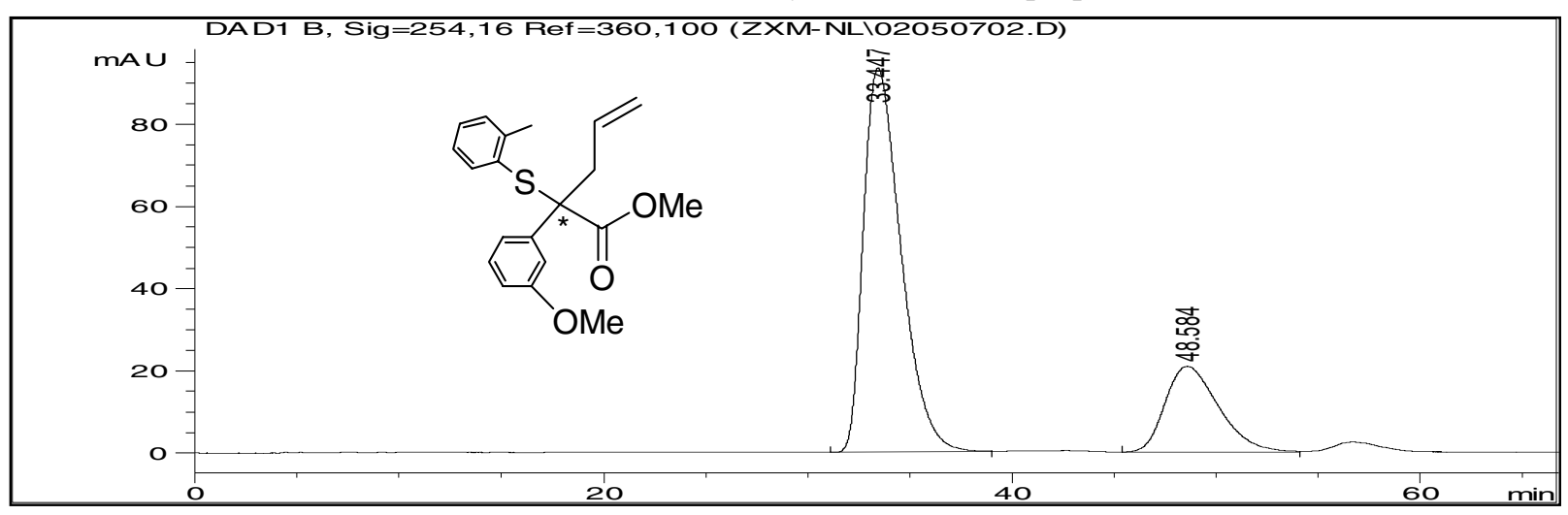

$A r^{\prime}=2-M e P h, A r=p-B r P h$, Racemic $\quad($ Hexane/iso-propanol $99: 1)$

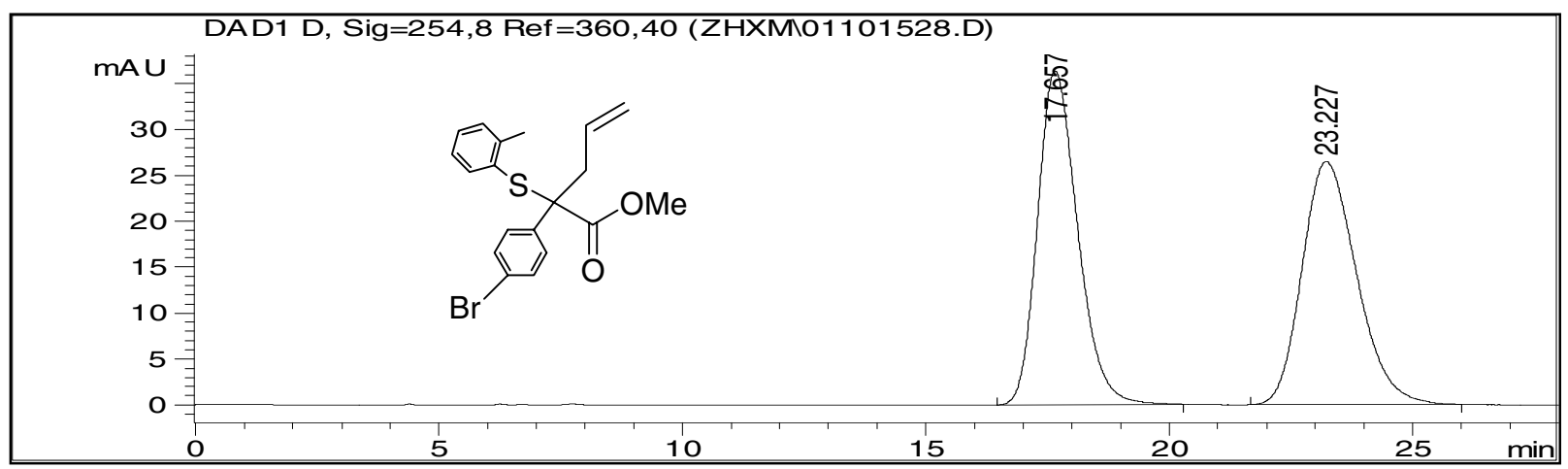

$A r^{\prime}=2-M e P h, A r=p-B r P h$, Table 2, Entry 6 (Hexane/iso-propanol 99 : 1)

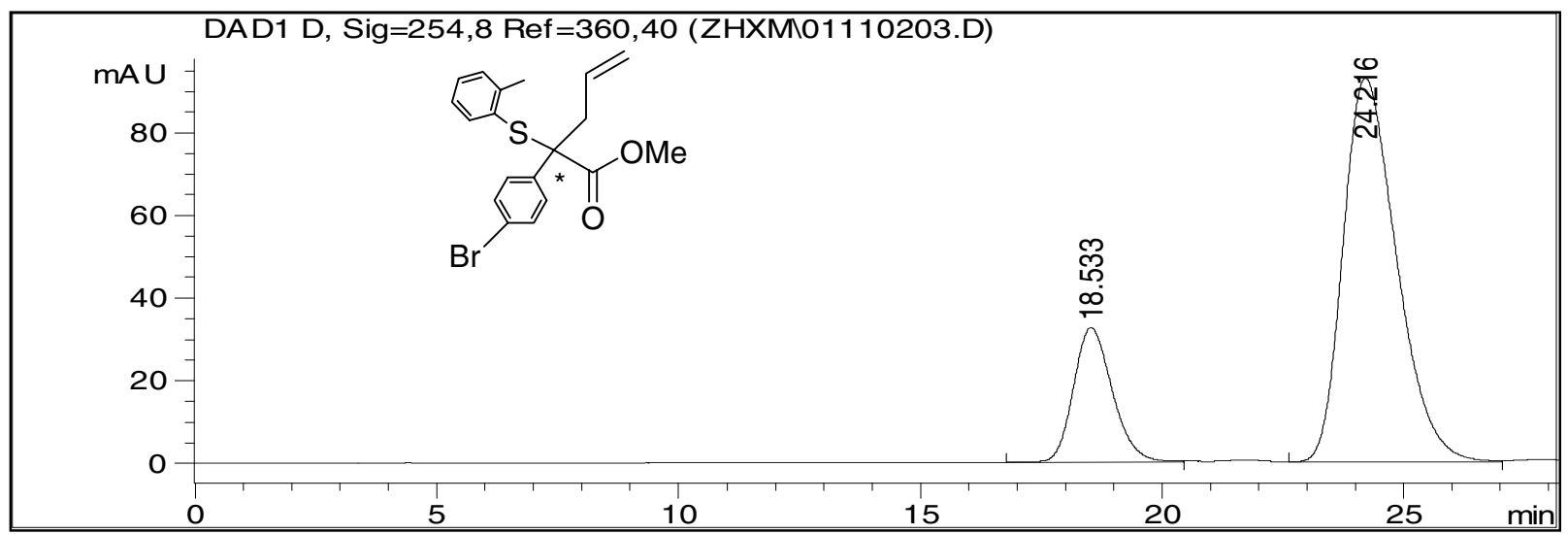


$A r^{\prime}=2-M e P h, A r=p-B r P h$, Table 2, Entry 7 (Hexane/iso-propanol $\left.99: 1\right)$

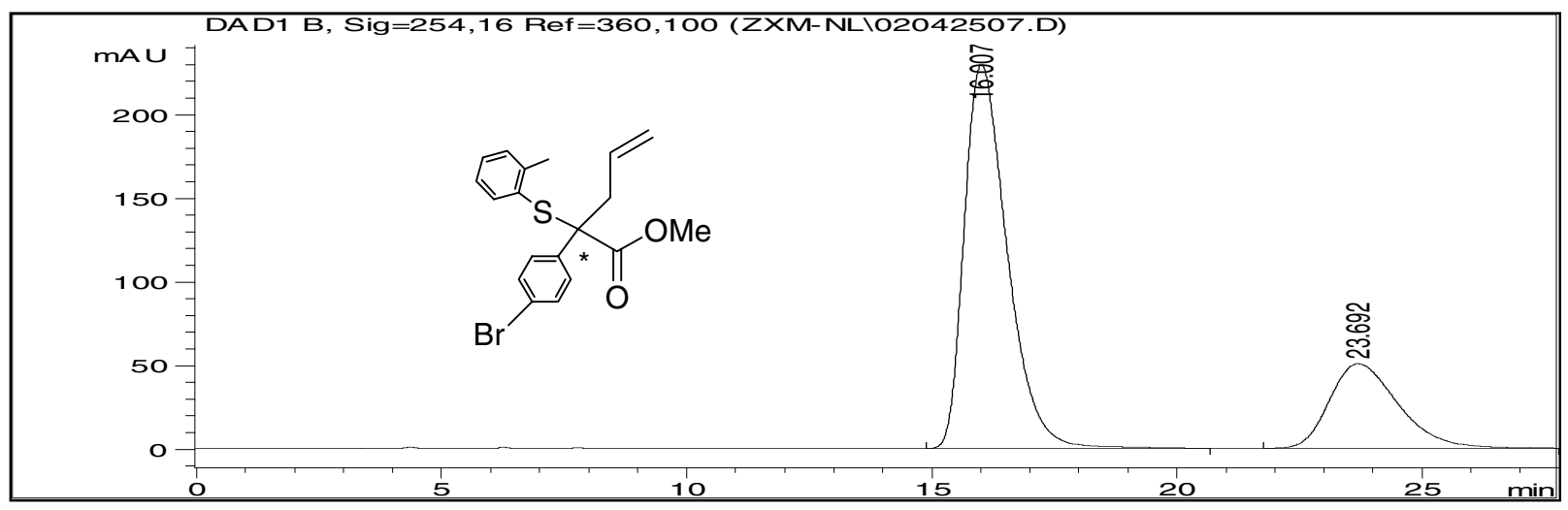

$A r^{\prime}=2-M e P h, A r=p-P h P h$, Racemic (Hexane/iso-propanol $\left.90: 10\right)$

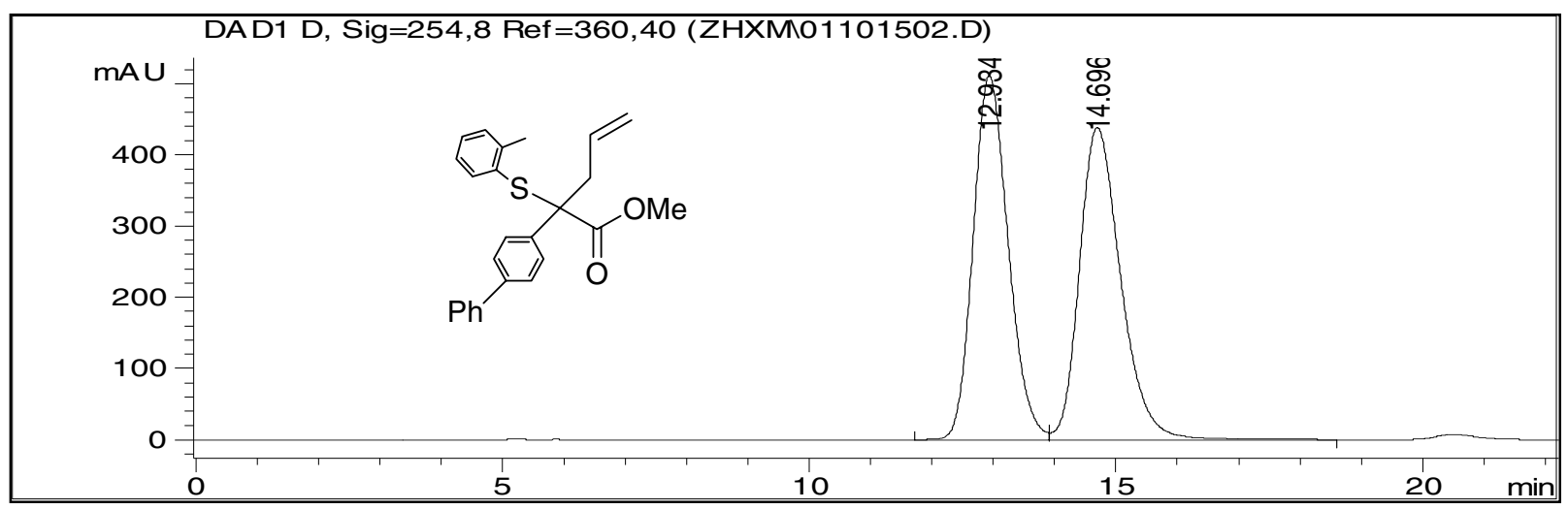

$A r^{\prime}=2-M e P h, A r=p-P h P h$, Table 2, Entry 8 (Hexane/iso-propanol $\left.90: 10\right)$

DAD1 D, Sig=254,8 Ref=360,40 (ZHXM01110206.D)

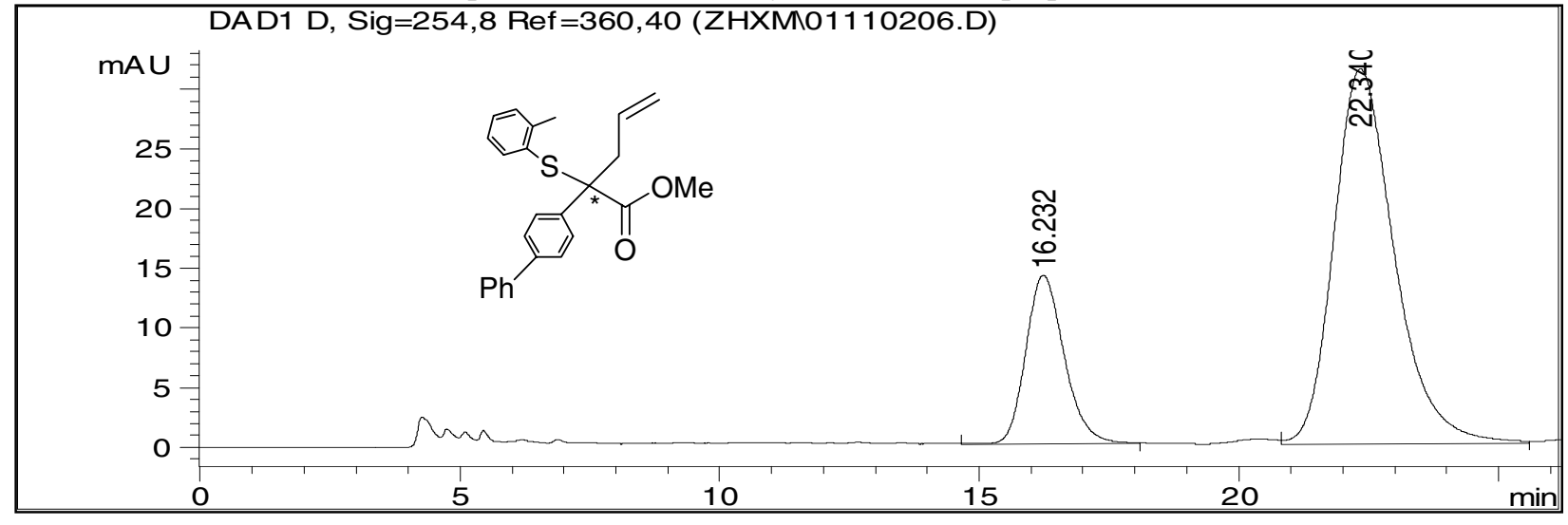


$A r^{\prime}=2-M e P h, A r=m-M e P h$, Racemic (Hexane/iso-propanol $99: 1$ )

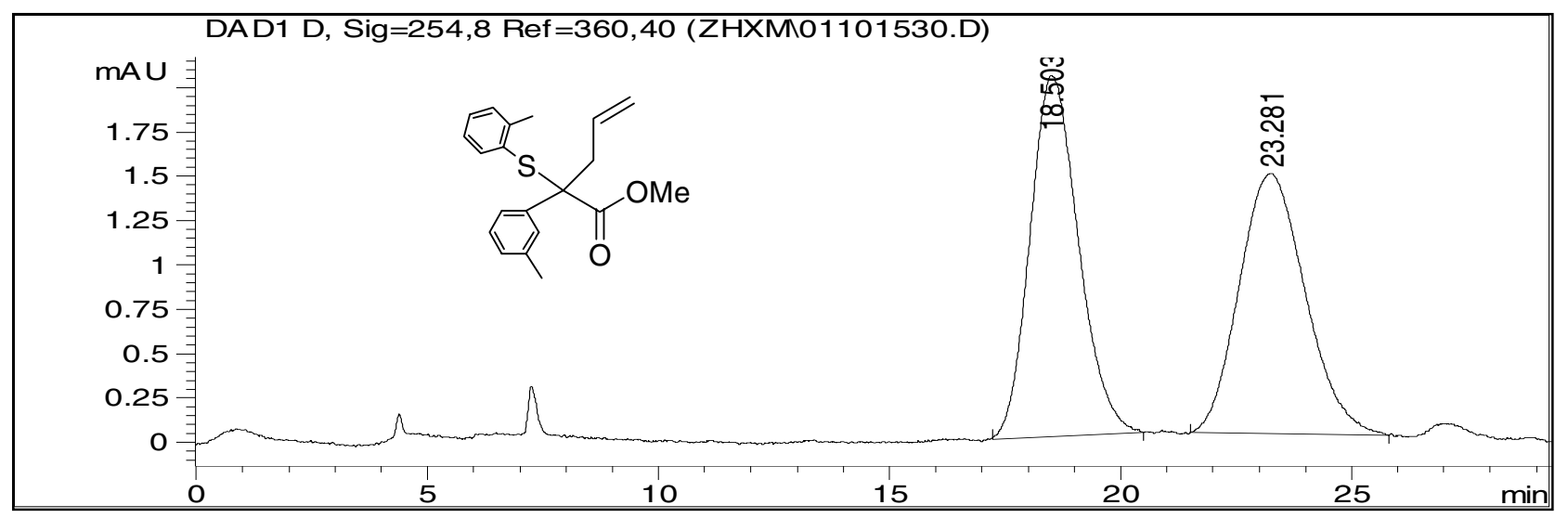

$A r^{\prime}=2-M e P h, A r=m-M e P h$, Table 2, Entry 9 (Hexane/iso-propanol $\left.99: 1\right)$

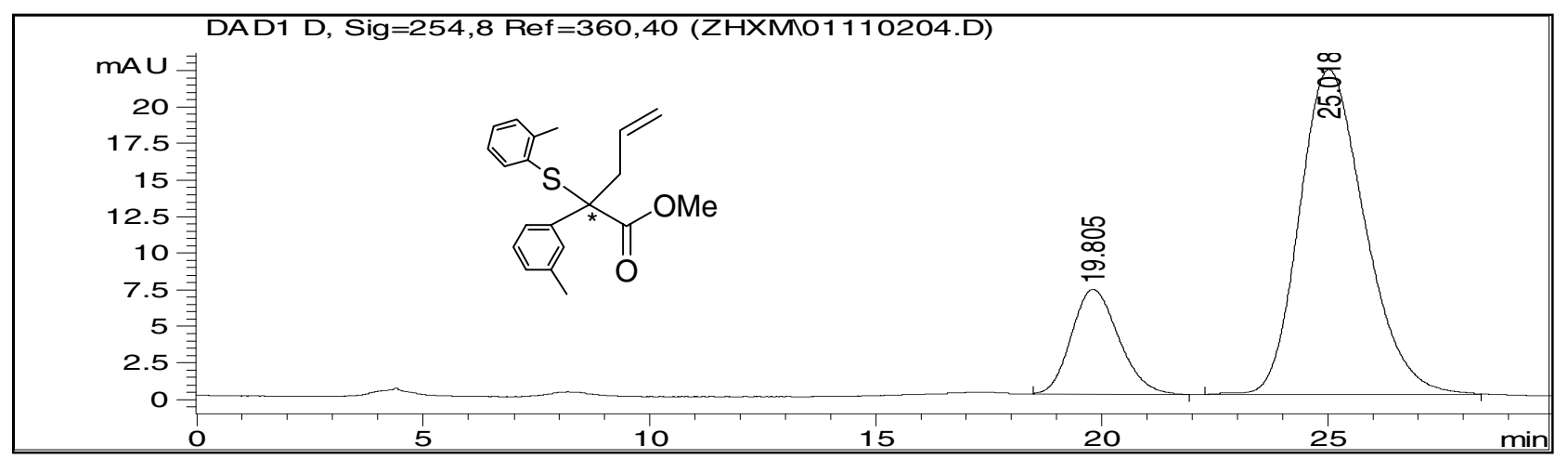

$A r^{\prime}=2-M e P h, A r=m-M e P h$, Table 2, Entry 10 (Hexane/iso-propanol $\left.99: 1\right)$

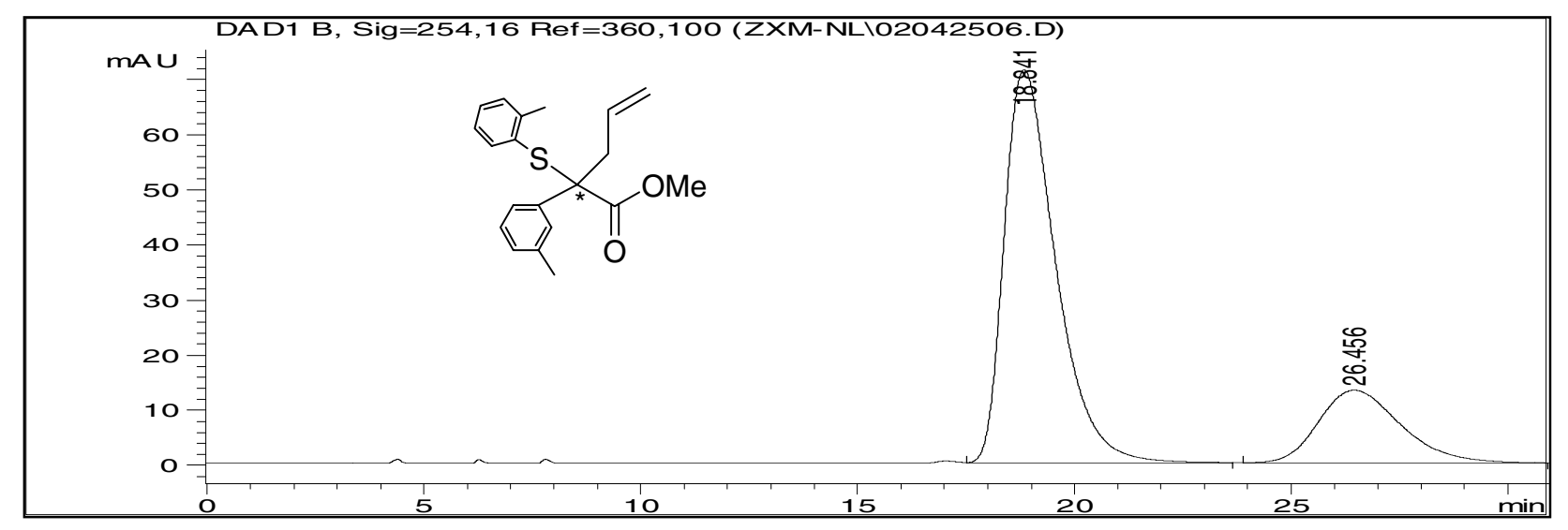


$A r^{\prime}=2-M e P h, A r=m-C l P h$, Racemic (Hexane/iso-propanol $90: 10$ )

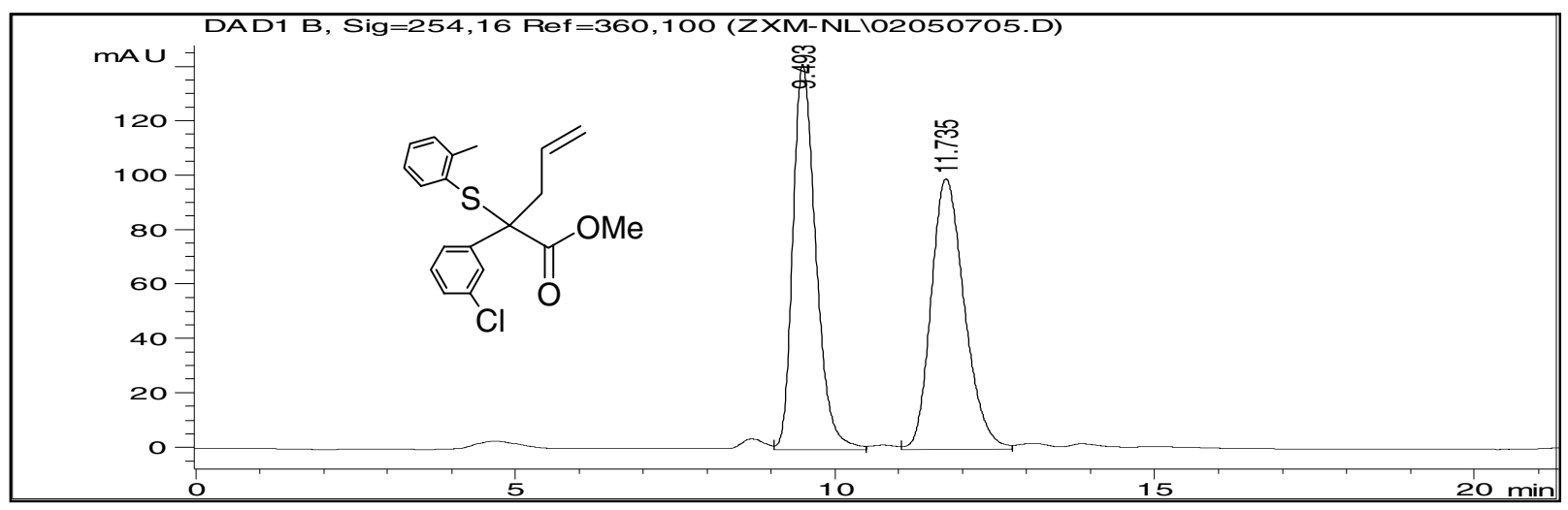

$A r^{\prime}=2-M e P h, A r=m-C l P h$, Table 2, Entry 11 (Hexane/iso-propanol $\left.90: 10\right)$

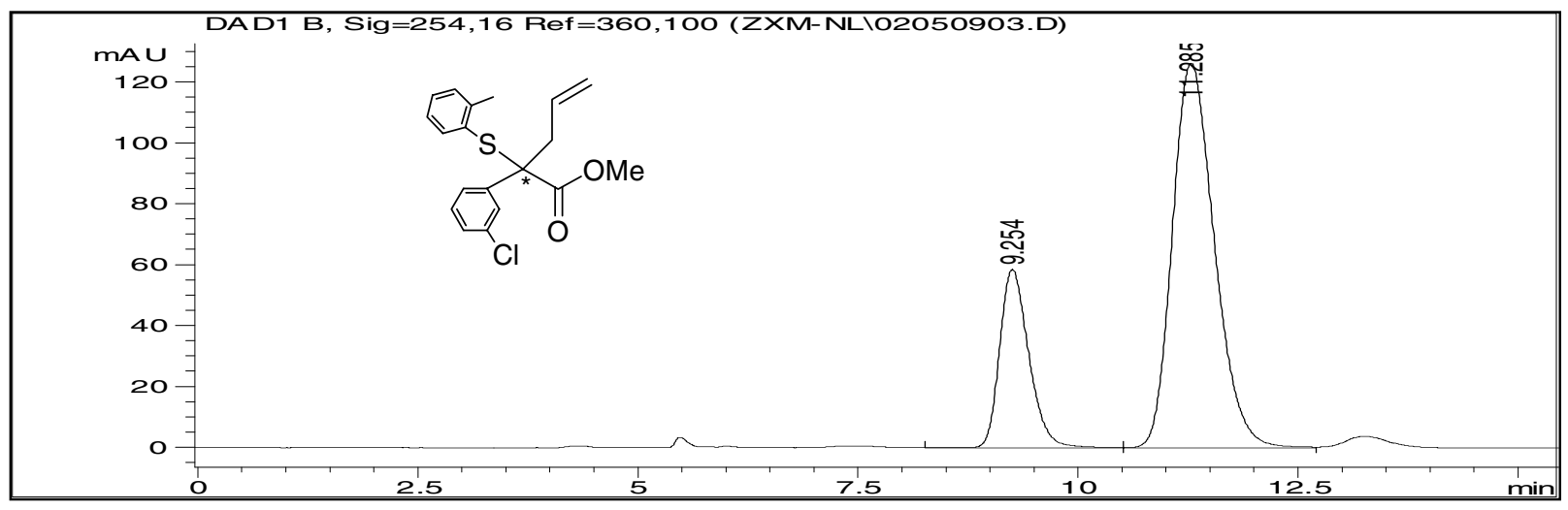

$A r^{\prime}=2-M e P h, A r=m-C l P h$, Table 2, Entry 12 (Hexane/iso-propanol $90: 10$ )

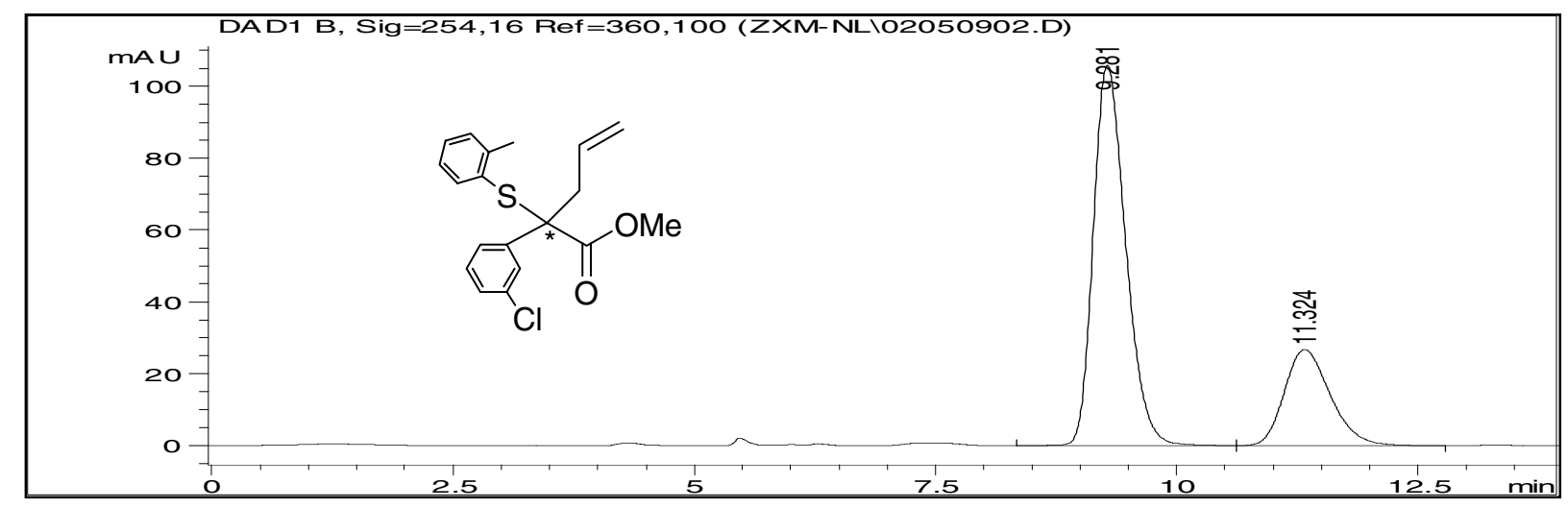


$A r^{\prime}=2-M e P h, A r=1-n a p h t h y l$, Racemic (Hexane/iso-propanol $95: 5$ )

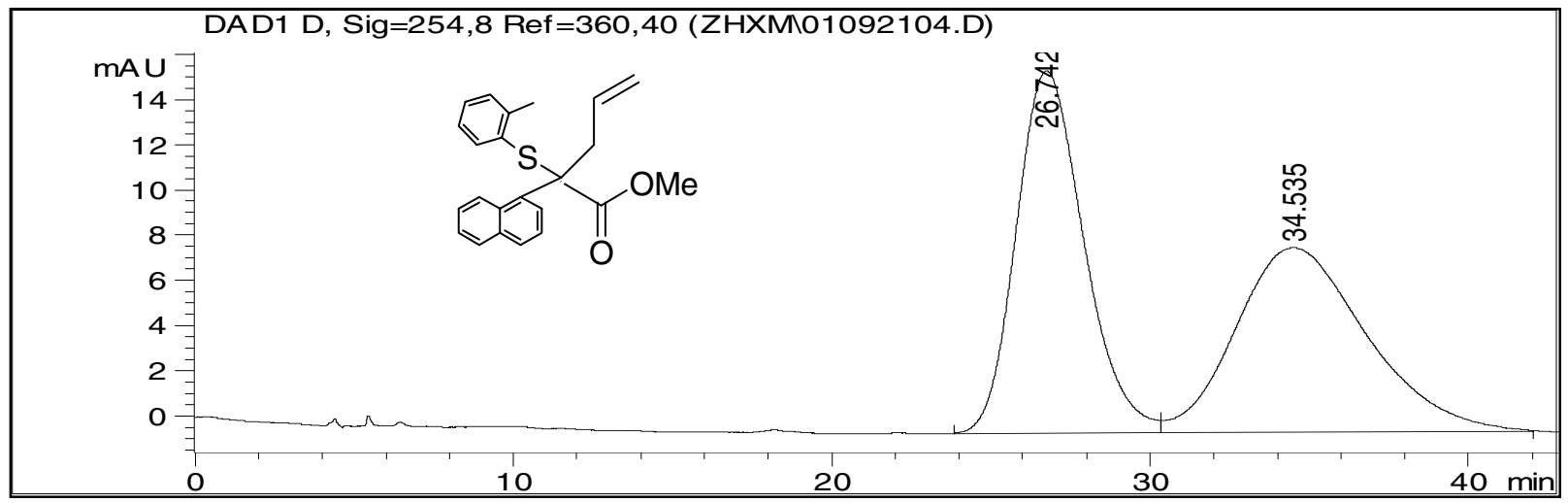

$A r^{\prime}=2-M e P h, A r=1-n a p h t h y l$, Table 2, Entry 13 (Hexane/iso-propanol $\left.95: 5\right)$

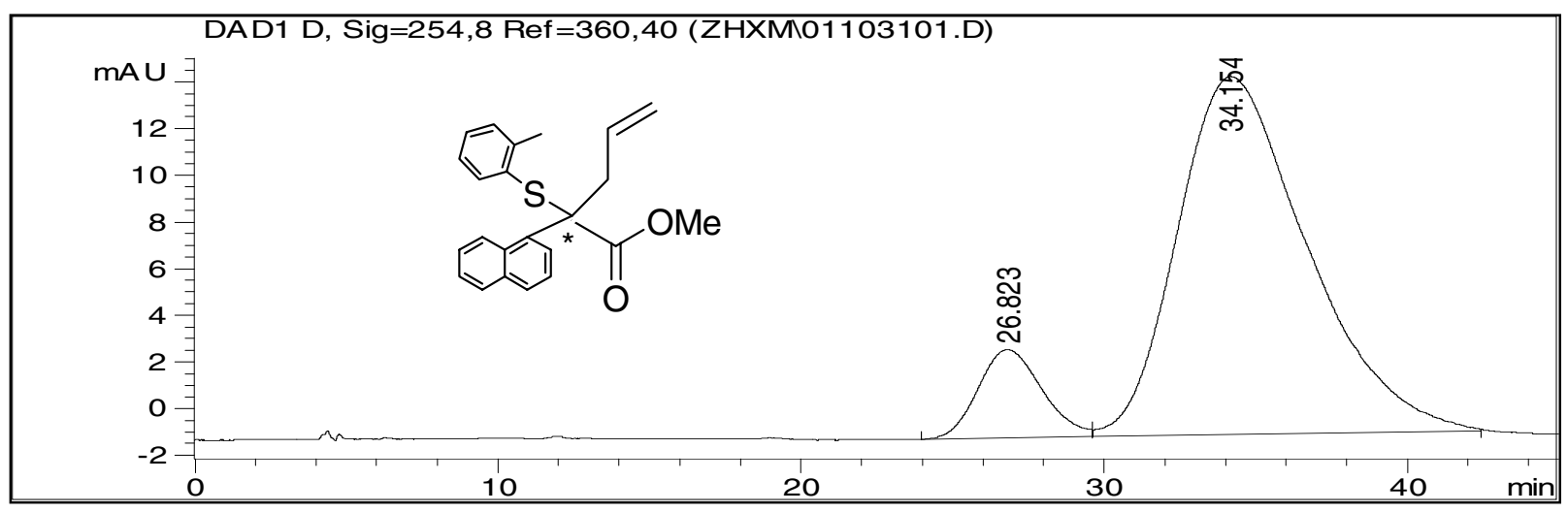

Ar' = 2-MePh, Ar = 1-naphthyl, Table 2, Entry 14 (Hexane/iso-propanol 95 : 5)

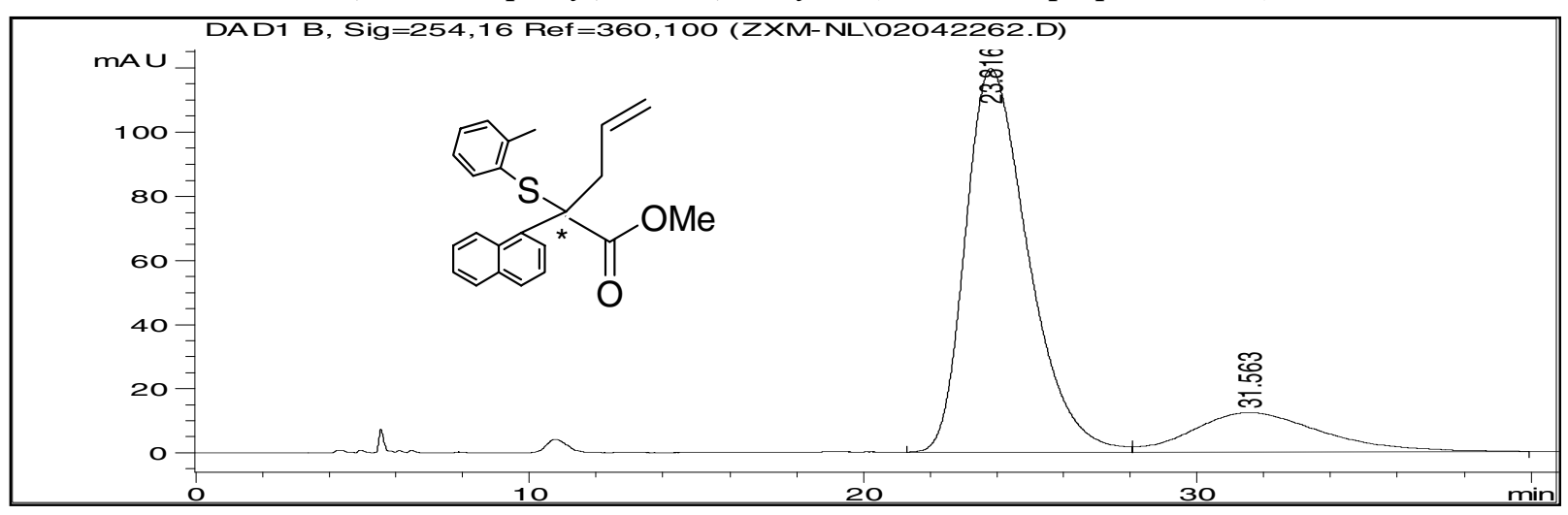


Ar' $=$ vinyl, $\boldsymbol{R}=\boldsymbol{P h}$, Racemic $($ Hexane/iso-propanol $90: 10)$

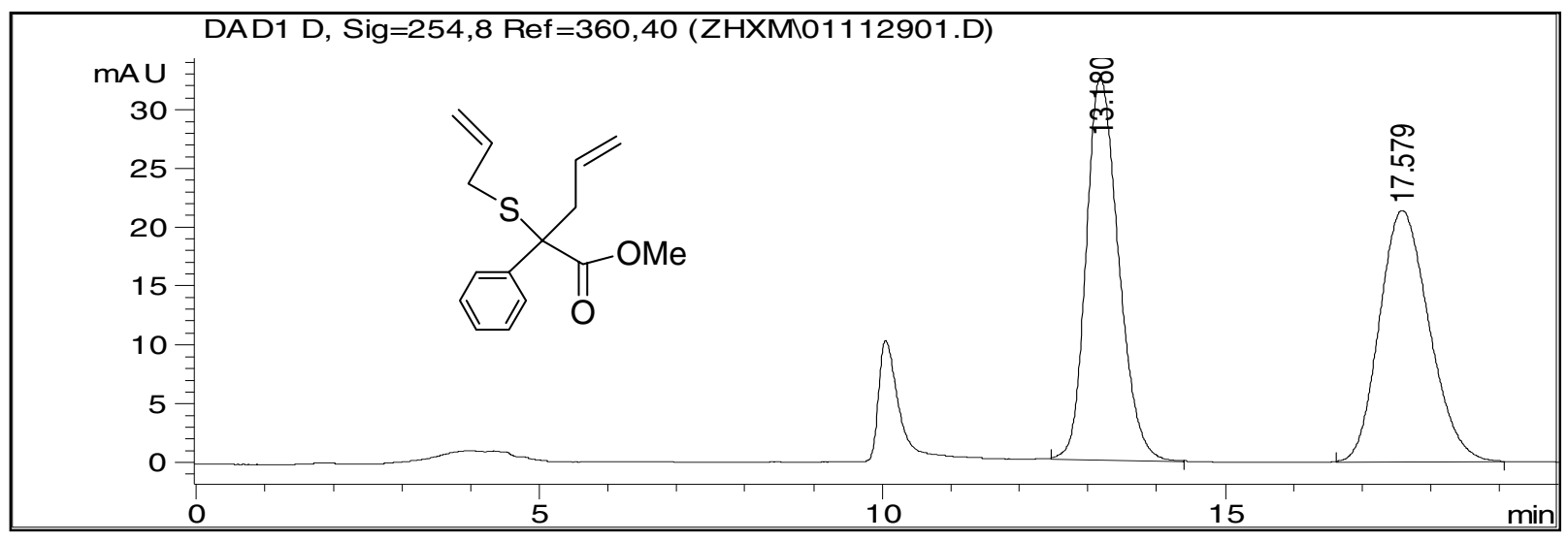

Scheme 4, compound 12 (Hexane/iso-propanol 90 : 10)

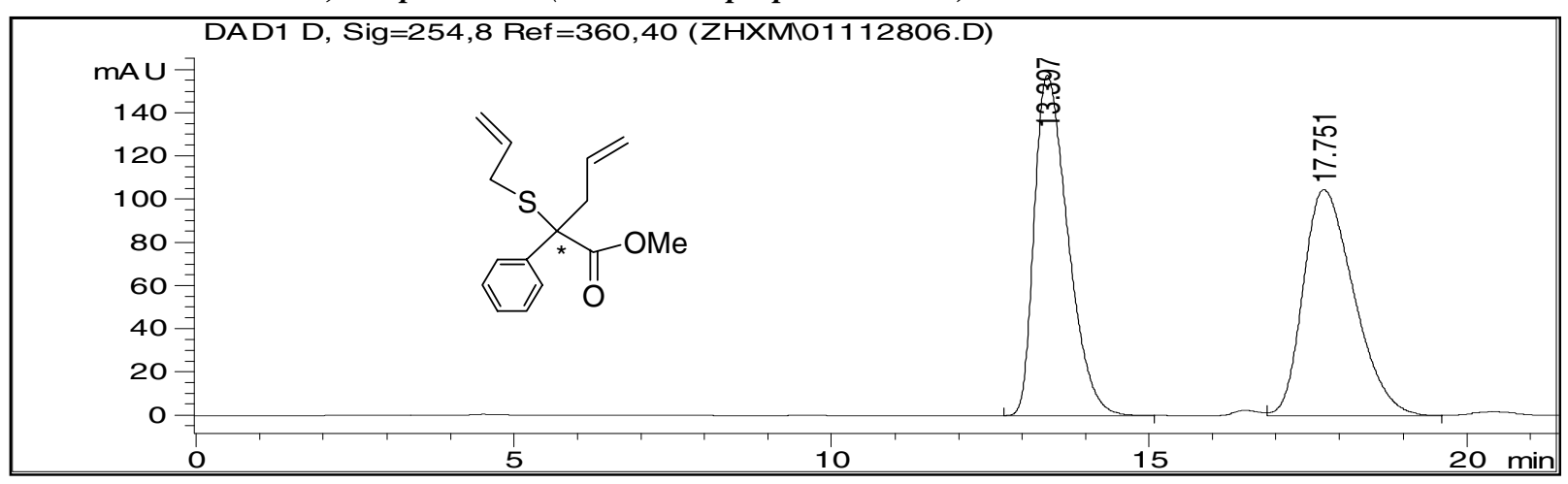


Figure 1. X-ray structure of $\mathbf{1 1}$

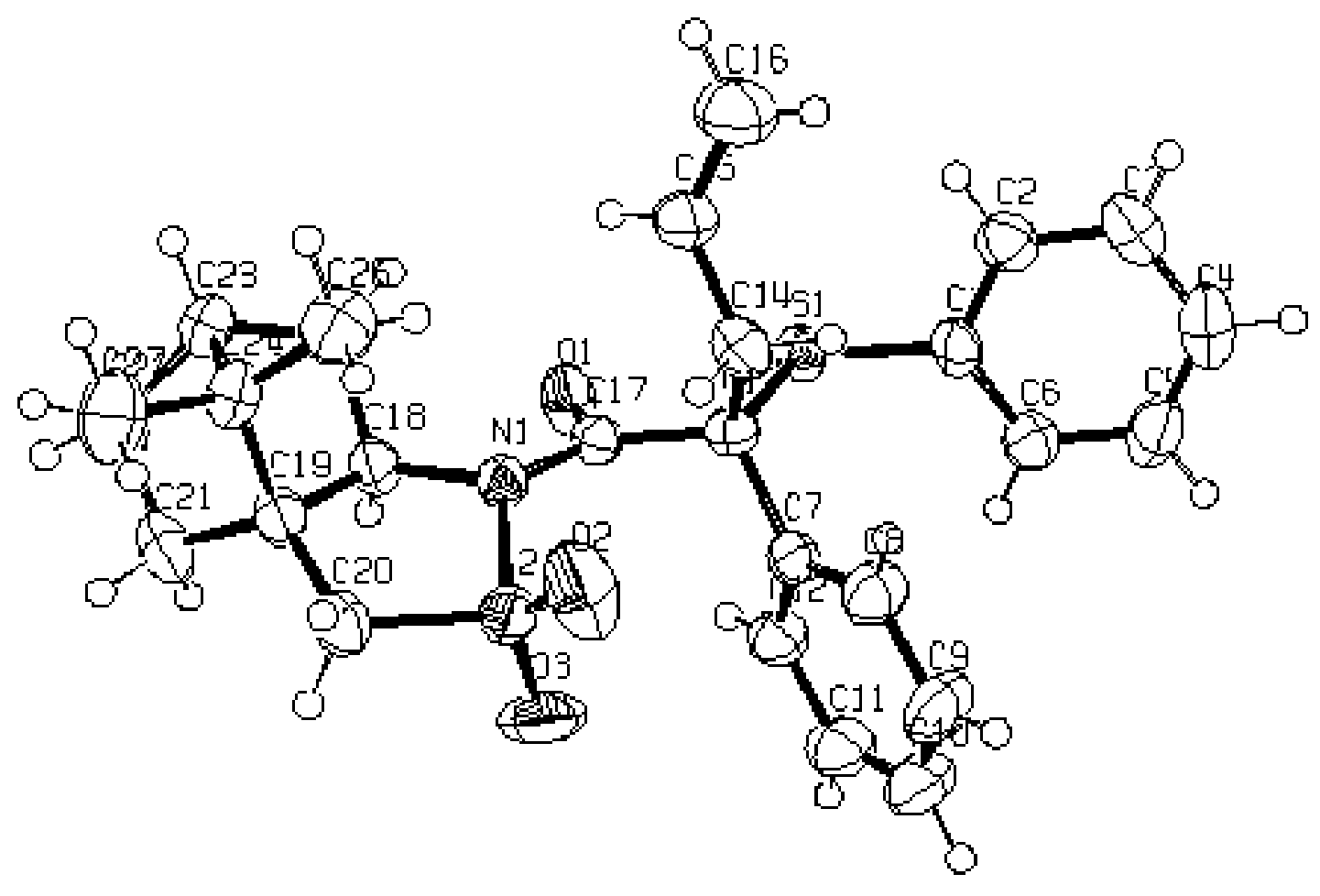

